\title{
Force/Motion Sliding Mode Control of Three Typical Mechanisms
}

\author{
Rong-Fong Fung ${ }^{1}$ and Chin-Fu Chang ${ }^{2}$ \\ ${ }^{1}$ Department of Mechanical \& Automation Engineering \\ National Kaohsiung First University of Science and Technology, \\ 1 University Road, Yenchau, Kaohsiung 824, \\ ${ }^{2}$ Institute of Engineering Science and Technology, \\ National Kaohsiung First University of Science and Technology, \\ 1 University Road, Yenchau, Kaohsiung 824,
}

Taiwan

\section{Introduction}

A number of papers [1-7] have been presented to address the issues of multi-body mechanisms. Examples of their applications are found in gasoline and diesel engines, where the gas force acts on the slider and the motion is transmitted through the links. Whether the connecting rod is assumed to be rigid or not, the steady-state and dynamic responses of the connecting rod of the mechanism with time-dependent boundary condition were obtained by Fung et al. [1-3]. In addition, a number of controllers, for example, repetitive control [4], adaptive control [5], computed torque control [6], and fuzzy neural network control [7] were designed for the multi-body mechanisms.

Over the past 25 years, the SMC algorithm [8-10] has been taken into account for dynamic control problems. The main feature of the SMC is to allow the sliding mode to occur on a prescribed switching surface, so that the system is only governed by the sliding equation and remains insensitive to a class of disturbances and parameter variations [8]. It is noted that the SMC is a robust control method and has been well established in pure motion control [9]. Afterwards, in order to eliminate the chattering phenomenon, which is commonly found in simulation of discontinuous SMC systems, and to simplify a hybrid numerical method that incorporates benefits of both SMC and differential algebraic equations, the (DAE) stabilization method was developed and successfully used to simulate constrained multi-body systems (MBS) whether under holonomic constraint or not [10]. However, the development of a control law which has been induced by a constrained force has not been adequately developed consistently in the previous studies. Su et al. [11] attempted to use the SMC for simultaneous position and force control on a constrained robot manipulator. They asserted that the control law, along with inclusion of the constraint force error in the definition of the sliding surface, produces an asymptotically stable force tracking error. However, Grabbe and Bridges [12] addressed their formulation as being a departure from the typical definition of a sliding surface, which is a linear differential equation in one tracking error variable [13], and the errors in the separate force control law and stability analysis were presented in [11]. Recently, Lian and Lin [14] have proposed a 
new sliding surface in terms of motion error and force error, and claimed that the errors in [11] are improved; therefore, the asymptotic stability of the motion-tracking error and forcetracking error can be ensured. However, Dixon and Zergeroglu [15] pointed out an error in the sliding mode control stability analysis of [14].

In this chapter, our intent is to improve the errors in $[11,14]$ and simplify the control design and stability proof for the three typical mechanisms, including the slider-crank mechanism, the quick-return mechanism and the toggle mechanism as shown in Figs. 1 3 respectively, which are not seen in any references addressing the force/motion SMC. Here, a separate sliding surface is proposed using the measurements of the angular position and speed of the crank, but the SMC algorithm is derived as well in a simple manner using only the force tracking error to construct the controller. In these schemes, the force tracking error is shown to be arbitrarily small by changing the force control feedback gain. Then, by exploiting the structure of its dynamics, the fundamental properties of the dynamics are obtained to facilitate controller design, whereby the asymptotic stability of motion tracking error in sliding surface and force tracking error accumulated in controller can be ensured.

The organization of this chapter is arranged as follows. In Section 2, the kinematic and dynamic analysis of the multi-body mechanism is investigated. A number of previous papers [4-7, 16-17] have shown the position and speed controllers for the regulation and tracking problems of the multi-body mechanism in the theoretical analysis and experimental results. However, control of the constrained force has not been investigated. The SMC laws are designed in Section 3. The simulated examples are shown in Section 4 and, finally, some conclusions are drawn.

\section{Dynamics analysis}

\subsection{Dynamic equation of motion}

Based on the Euler-Lagrange formulation [4], the equation of motion for a mechanism can be expressed as:

$$
\mathbf{M}(\mathbf{Q}) \ddot{Q}+\mathbf{N}(\mathbf{Q}, \dot{\mathrm{Q}})+\Phi_{\mathrm{Q}}^{\mathrm{T}} \boldsymbol{\lambda}=\mathbf{Q}^{\mathrm{A}}+\mathbf{U}
$$

where $\mathbf{M}(\mathbf{Q})$ is an $n \times n$ inertia matrix, $\mathbf{Q} \in R^{n}$ is the generalized coordinate vector, $\mathbf{N}(\mathbf{Q}, \dot{\mathbf{Q}}) \in R^{n}$ is the nonlinear vector, $\lambda \in R^{m}$ is the vector of Lagrange multipliers, $\boldsymbol{\Phi}_{\mathbf{Q}}=[\partial \boldsymbol{\Phi} / \partial \mathbf{Q}] \in R^{m \times n}$ is the partial derivative of the constraint equation with respect to the coordinate and is called the constraint Jacobian matrix, $\mathbf{Q}^{\mathbf{A}} \in R^{n}$ is the vector of nonconservative forces and $\mathbf{U} \in R^{n}$ is the vector of applied control efforts.

In order to obtain the general form of the force/motion controller design, we rewrite the nonlinear vector as:

$$
\mathbf{N}(\mathbf{Q}, \dot{\mathrm{Q}})=\mathbf{N}_{\mathrm{C}}(\mathbf{Q}, \dot{\mathrm{Q}}) \dot{\mathrm{Q}}+\mathbf{N}_{\mathrm{G}}(\mathbf{Q})
$$

where $\mathbf{N}_{\mathbf{C}}(\mathbf{Q}, \dot{\mathbf{Q}}) \in R^{n \times n}$ is the vector of coriolis and centrifugal forces; $\mathbf{N}_{\mathbf{G}}(\mathbf{Q}) \in R^{n}$ is the vector of gravitational force.

Then, Equation (1) becomes:

$$
\mathbf{M}(\mathbf{Q}) \ddot{\mathbf{Q}}+\mathbf{N}_{\mathrm{C}}(\mathbf{Q}, \dot{\mathbf{Q}}) \dot{\mathbf{Q}}+\mathbf{N}_{\mathrm{G}}(\mathbf{Q})=\mathbf{Q}^{\mathrm{A}}+\mathrm{U}+\mathbf{F} \text {. }
$$

where $\mathbf{F}=-\boldsymbol{\Phi}_{\mathrm{Q}}^{\mathrm{T}} \boldsymbol{\lambda}$ is the constraint force. 


\subsection{Dynamic properties of the mechanism}

Equation (3) is similar to the motion equation of an n-link rigid constrained robot $[11,15]$ in the state space. Two simplifying properties should be noted about this dynamic structure:

Property 1. The individual terms on the left-hand side of Equation (3) and the whole dynamics are linear in terms of a suitably selected set of equivalent manipulator and load parameters, i.e.,

$$
\mathbf{M}(\mathbf{Q}) \ddot{\mathbf{Q}}+\mathbf{N}_{\mathrm{C}}(\mathbf{Q}, \dot{\mathrm{Q}}) \dot{\mathbf{Q}}+\mathbf{N}_{\mathrm{G}}(\mathbf{Q})=\mathbf{Y}(\mathbf{Q}, \dot{\mathbf{Q}}, \ddot{\mathbf{Q}}) \alpha \text {. }
$$

where $\mathbf{Y}(\mathbf{Q}, \dot{\mathbf{Q}}, \ddot{\mathbf{Q}})$ is a $n \times r$ matrix; $\alpha \in R^{r}$ is the vector of equivalent parameters.

Property 2. From the given proper definition of the matrix $\mathbf{N}_{C}(Q, \dot{Q}), \dot{M}(Q)-2 \mathbf{N}_{C}(Q, \dot{Q})$ is skew-symmetric. The detailed proof can be seen in Appendix A.

Due to the presence of $m$ constraints, the degree of freedom of the mechanism is $(n-m)$. In this case, $(n-m)$ linearly independent coordinates are sufficient to characterize the constrained motion. From the implicit function theorem, the constraint Equation (1) can always be expressed as [18]:

$$
\mathbf{p}=\boldsymbol{\sigma}(\mathbf{q})
$$

Equation (5) is assumed that the elements of $\mathbf{q}$ are chosen to be the last (n-m) components of Q. If not the above case, Equation (1) still could always be reordered so that the last $(n-m)$ equations would correspond to $\mathbf{q}$ and the first $m$ equations to $\mathbf{p}$. That is, $\mathbf{Q}=\left[\begin{array}{ll}\mathbf{p}^{\mathbf{T}} & \mathbf{q}^{\mathbf{T}}\end{array}\right]^{\mathbf{T}}$.

Then, to simplify the equation form of the dynamic model, defining

$$
\mathbf{L}(\mathbf{q})=\frac{\partial \mathbf{Q}}{\partial \mathbf{q}}=\left[\begin{array}{ll}
\frac{\partial \mathbf{p}}{\partial \mathbf{q}} & \frac{\partial \mathbf{q}}{\partial \mathbf{q}}
\end{array}\right]^{T}=\left[\begin{array}{ll}
\frac{\partial \mathbf{\sigma}(\mathbf{q})}{\partial \mathbf{q}} & \mathbf{I}_{\mathbf{n}-\mathbf{m}}
\end{array}\right]^{T} \in R^{n \times(n-m)} .
$$

and using Equation (5), we have:

$$
\begin{gathered}
\dot{\mathbf{Q}}=\mathbf{L}(\mathbf{q}) \dot{\mathbf{q}} \\
\ddot{\mathbf{Q}}=\mathbf{L}(\mathbf{q}) \ddot{\mathbf{q}}+\dot{\mathbf{L}}(\mathbf{q}) \dot{\mathbf{q}}
\end{gathered}
$$

Therefore, the dynamic model of Equation (3) restricted to the constraint surface can be expressed in a reduced form as:

$$
\mathbf{M}(\mathbf{q}) \mathbf{L}(\mathbf{q}) \ddot{\mathbf{q}}+\mathbf{N}_{1}(\mathbf{q}, \dot{\mathbf{q}}) \dot{\mathbf{q}}+\mathbf{N}_{\mathrm{G}}(\mathbf{q})=\mathbf{Q}^{\mathrm{A}}+\mathbf{U}+\mathbf{F}
$$

where

$$
\mathbf{N}_{\mathbf{1}}(\mathbf{q}, \dot{\mathbf{q}})=\mathbf{M}(\mathbf{q}) \dot{\mathrm{L}}(\mathbf{q})+\mathbf{N}_{\mathbf{C}}(\mathbf{q}, \dot{\mathbf{q}}) \mathbf{L}(\mathbf{q})
$$

By exploiting the structure of Equation (9), three properties can be obtained as follows:

Property 3. In terms of a suitably selected set of parameters, the motion equation (9) is still linear, i.e.

$$
\mathbf{M}(\mathbf{q}) \mathbf{L}(\mathbf{q}) \ddot{\mathbf{q}}+\mathbf{N}_{1}(\mathbf{q}, \dot{\mathbf{q}}) \dot{\mathbf{q}}+\mathbf{N}_{\mathrm{G}}(\mathbf{q})=\mathbf{Y}_{1}(\mathbf{q}, \dot{\mathbf{q}}, \ddot{\mathbf{q}}) \alpha
$$


Property 4. Define the matrix as

$$
\mathbf{A}(\mathbf{q})=\mathbf{L}^{\mathbf{T}}(\mathbf{q}) \mathbf{M}(\mathbf{q}) \mathbf{L}(\mathbf{q}) \in R^{(n-m) \times(n-m)} .
$$

Then, $\dot{\mathbf{A}}=\mathbf{2} \mathbf{L}^{\mathrm{T}} \mathbf{( q )} \mathbf{N}_{\mathbf{1}}(\mathbf{q}, \dot{\mathbf{q}})$ is skew-symmetric, where $N_{1}(q, \dot{q})=M(q) \dot{L}(q)+N_{C}(q, \dot{q}) L(q) \in R^{n \times(n-m)}, L(q) \in R^{n \times(n-m)}$.

Property 5. $\left[\Phi_{\mathrm{Q}} \mathrm{L}(\mathrm{q})\right]^{\mathrm{T}}=\mathbf{L}^{\mathrm{T}}(\mathrm{q}) \Phi_{\mathrm{Q}}^{\mathrm{T}}=\mathbf{0}$.

The above three properties are basic principle in designing the force/motion SMC law.

\section{Design of the SMC Law}

\subsection{The sliding mode controller design}

A number of previous papers have only shown the position and speed controller designed for the regulation and tracking problems control of the constrained mechanisms. However, control of the constrained force has not been investigated in the previous studies. In this section, a separate sliding surface is proposed using the measurements of the angular position and speed of the crank, but the SMC algorithm is derived as well in a simple manner using only the force tracking error to construct the controller.

Given a desired trajectory $\mathbf{q}_{\mathbf{d}}$ and a desired constrained force $\mathbf{F}_{\mathbf{d}}$, or identically a desired multiplier $\lambda_{d}$, which satisfy the imposed constraint, i.e., $\boldsymbol{\Phi}\left(\mathbf{q}_{d}\right)=0$ and $\mathbf{F}_{d}=-\boldsymbol{\Phi}_{\mathbf{Q}}^{T}\left(\mathbf{q}_{d}\right) \lambda_{d}$. The control objective is to determine the SMC law such that $\mathbf{q} \rightarrow \mathbf{q}_{\mathbf{d}}$ and $\lambda \rightarrow \lambda_{\mathbf{d}}$ as $t \rightarrow \infty$.

From the SMC methodology, we define the tracking error $\mathbf{e}_{\mathbf{m}} \in R^{n-m}$ and a sliding surface $\mathbf{s}_{\mathbf{1}} \in R^{n-m}$ as:

$$
\begin{gathered}
\mathbf{e}_{\mathbf{m}}=\mathbf{q}(\mathbf{t})-\mathbf{q}_{\mathbf{d}}(\mathbf{t}) . \\
\mathbf{s}_{\mathbf{1}}=\dot{\mathbf{q}}-\dot{\mathbf{q}}_{\mathbf{r}}=\dot{\mathbf{e}}_{\mathbf{m}}+\Lambda \mathbf{e}_{m} .
\end{gathered}
$$

where $\mathbf{q}_{\mathbf{r}} \in R^{n-m}$ is the reference trajectory and $\Lambda \in R^{(n-m) \times(n-m)}$ is a tunable matrix.

The sliding controller [12] is defined as:

$$
\mathbf{U}=\mathbf{Y}_{1}(\mathbf{q}, \dot{\mathbf{q}}, \ddot{\mathbf{q}}) \varphi-\mathbf{L}(\mathbf{q}) \mathbf{s}_{1}+\Phi_{\mathbf{Q}}^{\mathrm{T}}(\mathbf{q}) \lambda_{\mathrm{c}}-\mathbf{Q}^{\mathrm{A}} .
$$

where $\mathbf{Y}_{\mathbf{1}}$ is a $n \times r$ matrix of known functions of $\mathbf{q}, \dot{\mathbf{q}}$ and $\ddot{\mathbf{q}}, \mathbf{L}(\mathbf{q})$ is defined in Equation (6), $\varphi=\left[\varphi_{1} \ldots \varphi_{\mathbf{r}}\right]^{T} \in R^{r}$ is the vector of switching functions, and $\lambda_{\mathrm{c}} \in R^{\mathrm{m}}$ is a force control that is defined as:

$$
\lambda_{\mathrm{c}}=\lambda_{\mathrm{d}}-\mathrm{Ke}_{\lambda} .
$$

where $\mathbf{K}$ is a $\mathrm{m} \times \mathrm{m}$ constant matrix of force control feedback gains, and $\mathbf{e}_{\mathbf{\lambda}}$ is the error vector of the multipliers and defined as

$$
\mathbf{e}_{\lambda}=\lambda-\lambda_{\mathrm{d}} \in R^{\mathrm{m}}
$$

\subsection{Stability analysis}

Substituting Equation (15) into the dynamic model of Equation (9), whose order was reduced using property 3 , we have: 


$$
\mathbf{Y}_{1}(\mathbf{q}, \dot{\mathbf{q}}, \ddot{\mathrm{q}}) \varphi-\mathbf{L}(\mathbf{q}) \mathbf{s}_{1}+\Phi_{\mathrm{Q}}^{\mathrm{T}}(\mathbf{q}) \lambda_{\mathrm{c}}-\Phi_{\mathrm{Q}}^{\mathrm{T}}(\mathbf{q}) \lambda=\mathbf{Y}_{1}(\mathbf{q}, \dot{\mathbf{q}}, \ddot{\mathbf{q}}) \alpha
$$

Defining $\alpha$ as a constant $r$-dimensional vector and replacing $\mathbf{q}$ by the reference trajectory $\mathbf{q}_{r}$, then the linear parameterization of the dynamics (Property 3 ) leads to:

$$
\mathbf{M}\left(\mathrm{q}_{\mathrm{r}}\right) \mathrm{L}\left(\mathrm{q}_{\mathrm{r}}\right) \ddot{\mathbf{q}}_{\mathrm{r}}+\mathbf{N}_{1}\left(\mathrm{q}_{\mathrm{r}}, \dot{\mathrm{q}}_{\mathrm{r}}\right) \dot{\mathrm{q}}_{\mathrm{r}}+\mathrm{N}_{\mathrm{G}}\left(\mathrm{q}_{\mathrm{r}}\right)=\mathrm{Y}_{1}\left(\mathrm{q}_{\mathrm{r}}, \dot{\mathrm{q}}_{\mathrm{r}}, \ddot{\mathrm{q}}_{\mathrm{r}}\right) \alpha .
$$

Using the derivative of the sliding surface equation (14) and substituting into Equation (11), we obtain:

$$
\operatorname{ML}\left(\dot{\mathbf{s}}_{1}+\ddot{\mathbf{q}}_{\mathrm{r}}\right)+\mathbf{N}_{\mathbf{1}}\left(\mathbf{s}_{1}+\dot{\mathbf{q}}_{\mathrm{r}}\right)+\mathbf{N}_{\mathrm{G}}=\mathrm{Y}_{1} \alpha .
$$

Then combining Equation (18) with Equation (20) and using Equation (19), we obtain:

$$
\mathrm{ML}_{1}=\mathrm{Y}_{1} \varphi-\mathrm{Y}_{1} \mathrm{a}-\mathbf{N}_{1} \mathbf{s}_{1}-\mathbf{L s}_{1}+\Phi_{\mathrm{Q}}^{\mathrm{T}} \lambda_{\mathrm{C}}-\Phi_{\mathrm{Q}}^{\mathrm{T}} \lambda .
$$

According to property 5, the above equation becomes:

$$
\begin{aligned}
\mathbf{A} \dot{s}_{1} & =\mathbf{L}^{\mathrm{T}} \mathbf{M L} \dot{s}_{1} \\
& =\mathbf{L}^{\mathrm{T}} \mathbf{Y}_{1} \varphi-\mathbf{L}^{\mathrm{T}} \mathbf{Y}_{1} \alpha-\mathbf{L}^{\mathrm{T}} \mathbf{N}_{\mathbf{1}} \mathbf{s}_{1}-\mathbf{L}^{\mathrm{T}} \mathbf{L} \mathbf{s}_{1} .
\end{aligned}
$$

To derive the control algorithm, the generalized Lyapunov function is considered as:

$$
\mathbf{V}=\frac{1}{2} \mathbf{s}_{1}^{\mathrm{T}} \mathbf{A s}_{\mathbf{1}}
$$

Differentiating $V$ with respect to time and using property 4, Equation (23) becomes:

$$
\begin{aligned}
\dot{\mathbf{V}} & =s_{1}{ }^{T} A \dot{s}_{1}+\frac{1}{2} s_{1}{ }^{T} \dot{A} s_{1}=s_{1}{ }^{T} A \dot{s}_{1}+s_{1}{ }^{T} L^{T} N_{1} s_{1} \\
& =s_{1}{ }^{T}\left(L^{T} Y_{1} \varphi-L^{T} Y_{1} \alpha-L^{T} N_{1} s_{1}-L^{T} L s_{1}\right)+s_{1}^{T} L^{T} N_{1} s_{1} \\
& =s_{1}{ }^{T}\left(L^{\mathrm{T}} Y_{1} \varphi-L^{\mathrm{T}} Y_{1} \alpha-L^{\mathrm{T}} L s_{1}\right) .
\end{aligned}
$$

The $\varphi$ is chosen as:

$$
\left.\varphi=-\bar{\alpha}_{\mathbf{1}} \mathbf{s g n}\left(\sum_{\mathbf{j}=\mathbf{1}}^{\mathbf{n}-\mathbf{m}} \mathbf{s}_{\mathbf{1} \mathbf{j}}\left(\mathbf{L}^{\mathrm{T}} \mathbf{Y}_{\mathbf{1}}\right)_{\mathbf{j i}}\right)\right) ; i=1,2, \ldots, r
$$

such that

$$
\dot{\mathbf{V}} \leq \mathbf{s}_{1}^{\mathrm{T}} \mathbf{L}^{\mathrm{T}} \mathbf{L} \mathbf{s}_{1}<0 .
$$

In the derivation of Eq. (24), it is noted that $\dot{M}(Q)-2 N_{C}(Q, \dot{Q})$ is skew-symmetric, and $L^{T}\left(\dot{M}-2 N_{C}\right) L$ is also skew-symmetric, which is the same as those in $[11,14]$. Besides, the special cases of the three typical mechanisms in this chapter, $L^{T}\left(\dot{M}-2 N_{C}\right) L$ is always equal to zero for $n-m=1$.

To reduce the chattering phenomenon along the sliding surface $s=0$, we adopt the quasilinear mode controller [13], which replaces the discontinuous term of sign function of 
Equation (25) with a continuous function inside a boundary layer around the sliding surface [24]. Therefore, the $\operatorname{sgn}(S)$ is replaced by the saturated function:

$$
\operatorname{sat}\left(\frac{s}{\mathcal{E}}\right)=\left\{\begin{array}{cc}
1 & \text { if } s>\mathcal{E}, \\
\frac{s}{\mathcal{E}} & \text { if }-\varepsilon<s<\mathcal{\varepsilon}, \\
-1 & \text { if } s>\mathcal{\varepsilon},
\end{array}\right.
$$

where $\varepsilon$ is the width of the boundary layer. This limits the tracking error and guarantees an accuracy of $\varepsilon$ order while alleviating the chattering phenomenon.

From Equation (23) and Equation (26), it is evident that a sliding surface $\left\|\mathbf{s}_{\mathbf{1}}\right\|$ is at last converged exponentially to zero, i.e., $\mathbf{e}_{\mathbf{m}} \rightarrow \mathbf{0}$ as $t \rightarrow \infty$. As if $\mathbf{q} \rightarrow \mathbf{q}_{\mathbf{d}}$, the condition $\mathbf{p}_{\mathbf{d}}=\boldsymbol{\sigma}\left(\mathbf{q}_{\mathbf{d}}\right)$ also implies that $\mathbf{p} \rightarrow \mathbf{p}_{\mathbf{d}}$.

Therefore,

$$
\mathbf{q} \rightarrow \mathbf{q}_{\mathbf{d}} \text { as } t \rightarrow \infty
$$

\section{Simulation examples of the three typical mechanisms}

\subsection{The slider-crank mechanism}

For more details on the kinematic and dynamic analysis of the slider-crank mechanism, refer to [19]. Using Hamilton's principle and Lagrange multipliers [20] and adopting the generalized coordinate vector $\mathbf{Q}=\left[\begin{array}{ll}\phi & \theta\end{array}\right]^{\mathrm{T}}$ in Equation (1) for the slider-crank mechanism shown in Figure 1, the dynamic equation can be obtained associated with the following matrices and elements:

$$
\begin{aligned}
& \mathbf{M}=\left[\begin{array}{ll}
A & E \\
E & B
\end{array}\right] \mathbf{N}_{\mathbf{C}}=\left[\begin{array}{cc}
K_{1} & K_{2} \\
P_{1} & P_{2}
\end{array}\right] \mathbf{N}_{\mathbf{G}}=\left[\begin{array}{c}
K_{3} \\
P_{3}
\end{array}\right] \\
& \boldsymbol{\Phi}_{\mathbf{Q}}=\left[\begin{array}{ll}
-l \cos \phi & r \cos \theta
\end{array}\right] \quad \mathbf{Q}^{\mathbf{A}}=\left[\begin{array}{l}
\left(F_{B}+F_{E}\right) l \sin \phi \\
\left(F_{B}+F_{E}\right) r \sin \theta
\end{array}\right] \quad \mathbf{U}=\left[\begin{array}{l}
u_{1} \\
u_{2}
\end{array}\right] \\
& A=-\frac{1}{3} m_{2} l^{2}-m_{B} l^{2} \sin ^{2} \phi \quad E=-\left(\frac{1}{2} m_{2}+m_{B}\right) r l \sin \theta \sin \phi \\
& B=-\frac{1}{2} m_{1} r^{2}-\left(m_{2}+m_{B}\right) r^{2} \sin ^{2} \theta \\
& K_{1}=-m_{B} l^{2} \dot{\phi} \sin \phi \cos \phi \quad K_{2}=-\left(\frac{1}{2} m_{2}+m_{B}\right) r l \dot{\theta} \cos \theta \sin \phi \quad K_{3}=-\frac{1}{2} m_{2} g l \cos \phi \\
& P_{1}=-\left(\frac{1}{2} m_{2}+m_{B}\right) r l \dot{\phi} \sin \theta \cos \phi \quad P_{2}=-\left(m_{2}+m_{B}\right) r^{2} \dot{\theta} \sin \theta \cos \theta \quad P_{3}=0
\end{aligned}
$$

where the dimensions of the slider-crank mechanism are $n=2, m=1$, and $r=1$ in the dynamic analysis. For the single degree-of-freedom slider-crank mechanism, only one constraint equation exists, which can be shown as:

$$
\Phi(Q)=r \sin \theta-l \sin \phi=0 \text {. }
$$


The position of the slider $\mathbf{B}$ can be expressed as:

$$
x_{B}=r \cos \theta+l \cos \phi .
$$

Substituting Equation (28) into Equation (29) yields:

$$
x_{B}=r \cos \theta+\left[l^{2}-r^{2} \sin ^{2} \theta\right]^{\frac{1}{2}}
$$

The angular displacement of the crank can be obtained as:

$$
\theta=\cos ^{-1}\left[\frac{x_{B}^{2}+r^{2}-l^{2}}{2 r x_{B}}\right] .
$$

The result can also be obtained if the cosine law is applied.

The Jacobian matrix of the constraint equation (28) is

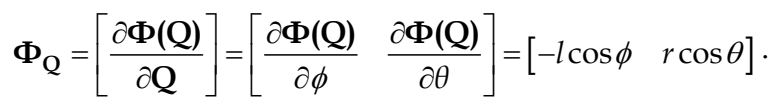

Differentiating Equation (28) with respect to time yields the constraint velocity equation:

$$
\dot{\Phi}(Q)=r \dot{\theta} \cos \theta-l \dot{\phi} \cos \phi=0 \text {. }
$$

Therefore, the matrix defined in Equation (6) becomes

$$
\mathbf{L}(\mathbf{q})=\frac{\partial \mathbf{Q}}{\partial \mathbf{q}}=\left[\begin{array}{ll}
\frac{\partial \phi}{\partial \theta} & \frac{\partial \theta}{\partial \theta}
\end{array}\right]^{T}=\left[\begin{array}{cc}
\frac{\dot{\phi}}{\dot{\theta}} & 1
\end{array}\right]^{T}=\left[\begin{array}{ll}
\frac{r \cos \theta}{l \cos \phi} & 1
\end{array}\right]^{T}
$$

and its first time derivative becomes

$$
\dot{\mathbf{L}}(\mathbf{q})=\left[\begin{array}{ll}
\frac{r l \dot{\phi} \sin \phi \cos \theta-r l \dot{\theta} \cos \phi \sin \theta}{l^{2} \cos ^{2} \phi} & 0
\end{array}\right]^{T}
$$

The dynamic equation (9) of the slider-crank mechanism, when restricted to the constraint equation (28), can be expressed as:

$$
\begin{aligned}
& {\left[\begin{array}{c}
A \frac{r \cos \theta}{l \cos \phi}+E \\
E \frac{r \cos \theta}{l \cos \phi}+B
\end{array}\right] \ddot{\theta}+\left[\begin{array}{c}
A \frac{r l \dot{\phi} \sin \phi \cos \theta-r l \dot{\theta} \cos \phi \sin \theta}{l^{2} \cos ^{2} \phi}+K_{1} \frac{r \cos \theta}{l \cos \phi}+K_{2} \\
E \frac{r l \dot{\phi} \sin \phi \cos \theta-r l \dot{\theta} \cos \phi \sin \theta}{l^{2} \cos ^{2} \phi}+P_{1} \frac{r \cos \theta}{l \cos \phi}+P_{2}
\end{array}\right] \dot{\theta}} \\
& +\left[\begin{array}{c}
-\frac{1}{2} m_{2} g l \cos \phi \\
0
\end{array}\right]=\left[\begin{array}{l}
\left(F_{B}+F_{E}\right) l \sin \phi \\
\left(F_{B}+F_{E}\right) r \sin \theta
\end{array}\right]+\left[\begin{array}{l}
u_{1} \\
u_{2}
\end{array}\right]+\left[\begin{array}{c}
l \cos \phi \\
-r \cos \theta
\end{array}\right] \lambda .
\end{aligned}
$$

The symbols $A, E, B, K_{1}, K_{2}, P_{1}, P_{2}$ and $P_{2}$ are shown in Equation (27). It is noted that $F_{B}$ is the friction force, $F_{E}$ is the external force, and $f_{1}=l \cos \phi \lambda$ and $f_{2}=-r \cos \theta \lambda$ are the constraint forces. From the results presented above, property 1 property 5 mentioned in Section 2.2 are all verified and fully satisfied in this example. 
The control objective is to design a feedback controller so that the angle $\theta$ tracks the desired trajectory $\theta_{d}$ and maintains the constraint force $\left[\begin{array}{ll}f_{1} & f_{2}\end{array}\right]^{T}$ to the desired one $\mathbf{F}_{\mathbf{d}}$. In here, $\theta_{d}$ and $\mathbf{F}_{\mathbf{d}}$ are assumed to be consistent with the imposed constraint. The block diagram of the SMC algorithm is shown in Figure 4.

Since $\lambda \rightarrow \lambda_{\mathbf{d}}$ means $\left[\begin{array}{ll}f_{1} & f_{2}\end{array}\right]^{T} \rightarrow \mathbf{F}_{\mathbf{d}}, \boldsymbol{\theta}_{\mathbf{d}}$ and $\boldsymbol{\lambda}_{\mathbf{d}}$ are chosen as $\boldsymbol{\theta}_{\mathbf{d}}=5.76(\mathrm{rad})$ and $\boldsymbol{\lambda}_{\mathbf{d}}=15$ in the simulations. The initial values of the constraint forces are assumed to be $\mathbf{F}_{\mathbf{d}}(\mathbf{0})=\left[\begin{array}{ll}\mathbf{f}_{\mathbf{1}}(\mathbf{0}) & \mathbf{f}_{\mathbf{2}}(\mathbf{0})\end{array}\right]^{\mathrm{T}}=\mathbf{0}$, i.e., $\boldsymbol{\lambda}(\mathbf{0})=\mathbf{0}$.

Using Equation (19), the applied control effort $\mathbf{U}=\left[\begin{array}{ll}u_{1} & u_{2}\end{array}\right]^{T}$ can be derived as:

$$
\mathbf{U}=\mathbf{Y}_{1} \varphi-\mathbf{L s}_{1}+\Phi_{\mathbf{Q}}^{\mathrm{T}} \lambda_{\mathrm{C}}-\mathbf{Q}^{\mathrm{A}}
$$

where

$$
\mathbf{Y}_{1}=\frac{1}{\mathbf{a}}\left[\mathbf{M L} \ddot{\theta}_{\mathrm{d}}+\mathbf{N}_{1} \dot{\boldsymbol{\theta}}_{\mathrm{d}}+\mathbf{N}_{\mathrm{G}}\right], \varphi=\left\{\begin{array}{c}
\overline{\mathbf{\alpha}}_{1}, \mathrm{~s}_{1}\left(\mathrm{~L}^{\mathrm{T}} \mathbf{Y}_{1}\right)<0 \\
-\overline{\mathbf{\alpha}}_{1}, \mathrm{~s}_{1}\left(\mathrm{~L}^{\mathrm{T}} \mathbf{Y}_{1}\right)>0
\end{array}, \mathbf{s}_{1}=\dot{\mathbf{e}}_{\mathrm{m}}+\Lambda \mathbf{e}_{\mathrm{m}}=\left(\dot{\boldsymbol{\theta}}-\dot{\boldsymbol{\theta}}_{\mathrm{d}}\right)+\Lambda\left(\boldsymbol{\theta}-\boldsymbol{\theta}_{\mathrm{d}}\right)\right.
$$

For numerical simulations, the parameters of the slider-crank mechanism are chosen as: $m_{1}=3.64 \mathrm{~kg}, m_{2}=1.18 \mathrm{~kg}, m_{B}=1.8 \mathrm{~kg}, r=0.1 \mathrm{~m}, l=0.305 \mathrm{~m}, \mathrm{lp}=0.055 \mathrm{~m}$. and $\alpha_{1}=1, \bar{\alpha}_{1}=1$ and $\Lambda=5$.

Since the trajectory tracking on the constraint surface with a specified constraint force is of interest, the initial position and speed of the slider-crank mechanism are chosen on the desired trajectory as:

$$
\begin{aligned}
& \theta(0)=4.712(\mathrm{rad}) ; \quad \phi(0)=-0.334(\mathrm{rad}) ; \quad \dot{\theta}(0)=0 ; \quad \dot{\phi}(0)=0 ; \\
& x_{B}\left(t_{0}\right)=0.343 \mathrm{~m}, x_{B}\left(t_{f}\right)=0.443 \mathrm{~m} .
\end{aligned}
$$

All the parameters in the SMC controller are chosen to achieve the best transient performance in numerical simulations under the limitation of the control effort and the requirements of stability. Furthermore, for the reason of using a single input actuation on joint 1 , the control effort is only needed in the second equation of the constrained motion of Equation (36). As to the first part of Equation (36), it shows that the force equilibrium either with holonomic or nonholonomic constraints and the torque is exerted at joint 2 . The responses of the crank angle, which is shown in Figure 5(a), reach the desired value in about $0.8 \mathrm{sec}$. The slider position manipulated from Equation (29) is shown in Figure $5(\mathrm{~b})$. The tracking results of the crank angle $\theta$ and the slider position $x_{B}$ coincide with previous studies by Fung et al. [16, 17]. The control effort of the applied torque $\tau$ is shown in Figure $5(c)$ and the sliding surface $s_{1}$ is shown in Figure 5(d). The Lagrange multiplier $\lambda_{C}$ is shown in Figure 6(a), and constraint forces of joints 1 and 2 are shown in Figure 6(b) and Figure 6(c), respectively. From Figures 5 and 6, the control objectives of force/motion of the slider-crank mechanism are achieved successfully.

\subsection{The quick-return mechanism}

To present the robustness and a well-established control method of the SMC controller, the quick-return and toggle mechanisms (see Section 4.3) will be chosen to verify the SMC algorithm, which is then adequately developed to the general case of multi-body mechanisms. The quick-return mechanism is addressed first, where the kinematic and 
dynamic analysis of the mechanism is found in [21] and the generalized coordinate vector $\mathbf{Q}=\left[\begin{array}{lll}\phi & \beta & \theta\end{array}\right]^{\mathrm{T}}$ in Equation (1) for the quick-return mechanism shown in Figure 2 is adopted. The dynamic equation can be obtained and is associated with the following matrices and elements:

$$
\begin{aligned}
& \mathbf{M}=\left[\begin{array}{ccc}
A 1 & G 1 & 0 \\
H 1 & B 1 & 0 \\
0 & 0 & C C
\end{array}\right] \mathbf{N}_{C}=\left[\begin{array}{ccc}
P 1 & T 1 & 0 \\
Y 1 & R 1 & 0 \\
0 & 0 & 0
\end{array}\right] \mathbf{N}_{\mathbf{G}}=0 \\
& \boldsymbol{\Phi}_{\mathbf{Q}}=\left[\begin{array}{ccc}
\cos \phi(D+R \cos \theta)+R \sin \theta \sin \phi & 0 & -R \sin \phi \sin \theta-R \cos \theta \cos \phi \\
-L \sin \phi & S \cos \beta & 0
\end{array}\right] \\
& \mathbf{Q}^{\mathbf{A}}=\left[\begin{array}{c}
P L \cos \phi \\
P S \sin \beta \\
0
\end{array}\right] \mathbf{U}=\left[\begin{array}{l}
u_{1} \\
u_{2} \\
u_{3}
\end{array}\right] \\
& A 1=-\frac{1}{3} m_{1} L^{2}-\left(m_{2}+m_{C}\right) L^{2} \cos ^{2} \phi, G 1=-\left(\frac{1}{2} m_{2}+m_{C}\right) S L \sin \beta \cos \phi, \\
& H 1=-\left(\frac{1}{2} m_{2}+m_{C}\right) S L \sin \beta \cos \phi, B 1=-\left(\frac{1}{3} m_{2}+m_{C} \sin ^{2} \beta\right) S^{2}, \\
& C C=-\frac{1}{3} m_{3} R^{2}, \quad P 1=\left(m_{2}+m_{C}\right) L^{2} \dot{\phi} \cos \phi \sin \phi, ~, \\
& T 1=-\left(\frac{1}{2} m_{2}+m_{C}\right) S L \dot{\beta} \cos \beta \cos \phi, Y 1=\left(\frac{1}{2} m_{2}+m_{C}\right) S L \dot{\phi} \sin \beta \sin \phi, \\
& R 1=-m_{C} S^{2} \dot{\beta} \sin \beta \cos \beta .
\end{aligned}
$$

where $n=3, m=2$ and $r=1$ are employed in the dynamic analysis.

For the single degree-of-freedom quick-return mechanism, there exist two constraint equations as follows:

$$
\boldsymbol{\Phi}(\mathbf{Q})=\left[\begin{array}{c}
\sin \phi(D+R \cos \theta)-R \sin \theta \cos \phi \\
S \sin \beta-L(1-\cos \phi)
\end{array}\right]=0 .
$$

where $\varphi$ can be obtained by analyzing its geometric relations

$$
\phi=\tan ^{-1} \frac{R \sin \theta}{D+R \cos \theta} .
$$

The position of slider $\mathrm{C}$ can be expressed as:

$$
x_{\mathrm{C}}=S \cos \beta-L \sin \phi .
$$

The Jacobian matrix of the constraint equations is: 


$$
\boldsymbol{\Phi}_{\mathrm{Q}}=\left[\begin{array}{ccc}
\cos \phi(D+R \cos \theta)+R \sin \theta \sin \phi & 0 & -R \sin \phi \sin \theta-R \cos \theta \cos \phi \\
-L \sin \phi & S \cos \beta & 0
\end{array}\right] .
$$

Therefore, the matrix defined in Equation (6) is:

$$
\begin{aligned}
& \mathbf{L}(\mathbf{q})=\frac{\partial \mathbf{Q}}{\partial \mathbf{q}}=\left[\begin{array}{lll}
\frac{\partial \phi}{\partial \theta} & \frac{\partial \beta}{\partial \theta} & \frac{\partial \theta}{\partial \theta}
\end{array}\right]^{T}=\left[\begin{array}{lll}
\frac{\dot{\phi}}{\dot{\theta}} & \frac{\dot{\beta}}{\dot{\theta}} & 1
\end{array}\right]=\left[\begin{array}{lll}
\frac{\dot{\phi}}{\dot{\theta}} & \frac{\dot{\beta}}{\dot{\phi}} \times \frac{\dot{\phi}}{\dot{\theta}} & 1
\end{array}\right] \\
& =\left[\begin{array}{lll}
\frac{\left(D R \cos \theta+R^{2}\right)}{D^{2}+R^{2}+2 D R \cos \theta} & \frac{L \sin \phi\left(D R \cos \theta+R^{2}\right)}{S \cos \beta\left(D^{2}+R^{2}+2 D R \cos \theta\right)} & 1
\end{array}\right]^{T}
\end{aligned}
$$

Then, differentiating Equation (43) with respect to time yields:

$$
\dot{\mathbf{L}}(\mathbf{q})=\left[\begin{array}{c}
\frac{D R \dot{\theta} \sin \theta\left(R^{2}-D^{2}\right)}{\left(D^{2}+R^{2}+2 D R \cos \theta\right)^{2}} \\
\frac{L S D R \dot{\theta} \sin \phi \sin \theta \cos \beta\left(R^{2}-D^{2}\right)}{S^{2} \cos ^{2} \beta\left(D^{2}+R^{2}+2 D R \cos \theta\right)^{2}}+ \\
\frac{L S\left(D^{2}+R^{2}+2 D R \cos \theta\right)\left(D R \cos \theta+R^{2}\right)(\dot{\beta} \sin \phi \sin \beta+\dot{\phi} \cos \phi \cos \beta)}{S^{2} \cos ^{2} \beta\left(D^{2}+R^{2}+2 D R \cos \theta\right)^{2}} \\
0
\end{array}\right]=\left[\begin{array}{c}
\dot{L}_{11} \\
\dot{L}_{21} \\
0
\end{array}\right] .
$$

The dynamic equation of a quick-return mechanism, when restricted to the constraint equation, can be expressed as:

$$
\begin{aligned}
& \left.\begin{array}{c}
A 1 \times\left\{\frac{\left(D R \cos \theta+R^{2}\right)}{D^{2}+R^{2}+2 D R \cos \theta}\right\}+G 1 \times\left\{\frac{L \sin \phi\left(D R \cos \theta+R^{2}\right)}{S \cos \beta\left(D^{2}+R^{2}+2 D R \cos \theta\right)}\right\} \\
H 1 \times\left\{\frac{\left(D R \cos \theta+R^{2}\right)}{D^{2}+R^{2}+2 D R \cos \theta}\right\}+B 1 \times\left\{\frac{L \sin \phi\left(D R \cos \theta+R^{2}\right)}{S \cos \beta\left(D^{2}+R^{2}+2 D R \cos \theta\right)}\right\}
\end{array}\right] \ddot{\theta}_{1}+ \\
& +\left[\begin{array}{c}
A 1 \times \dot{L}_{11}+G 1 \times \dot{L}_{21}+\frac{P 1 \times\left(D R \cos \theta+R^{2}\right)}{D^{2}+R^{2}+2 D R \cos \theta}+\frac{T 1 \times L \sin \phi\left(D R \cos \theta+R^{2}\right)}{S \cos \beta\left(D^{2}+R^{2}+2 D R \cos \theta\right)} \\
H 1 \times \dot{L}_{11}+B 1 \times \dot{L}_{21}+\frac{Y 1 \times\left(D R \cos \theta+R^{2}\right)}{D^{2}+R^{2}+2 D R \cos \theta}+\frac{R 1 \times L \sin \phi\left(D R \cos \theta+R^{2}\right)}{S \cos \beta\left(D^{2}+R^{2}+2 D R \cos \theta\right)} \\
0
\end{array}\right] \dot{\theta}_{1}= \\
& =\left[\begin{array}{c}
P L \cos \phi \\
P S \sin \beta \\
0
\end{array}\right]+\left[\begin{array}{l}
u_{1} \\
u_{2} \\
u_{3}
\end{array}\right]+\left[\begin{array}{cc}
\cos \phi(D+R \cos \theta)+R \sin \theta \sin \phi & -L \sin \phi \\
0 & S \cos \beta \\
-R \sin \phi \sin \theta-R \cos \theta \cos \phi & 0
\end{array}\right]\left[\begin{array}{l}
\lambda_{1} \\
\lambda_{2}
\end{array}\right] .
\end{aligned}
$$


The parameters $A 1, G 1, B 1, H 1, C C, \dot{L}_{11}, \dot{L}_{21}, P 1, T 1, Y 1, R 1$ are shown in Equation (38). It is noted that $P$ is the cutting force acting on the slider $C, \tau$ is the external force acting on rod 3 , and the constraint forces are expressed as:

$$
\begin{gathered}
f_{1}=\{\cos \phi(D+R \cos \theta)+R \sin \theta \sin \phi\} \lambda_{1}-L \lambda_{2} \sin \phi, \\
f_{2}=S \cos \beta \lambda_{2}, \\
f_{3}=\{-R \sin \phi \sin \theta-R \cos \theta \cos \phi\} \lambda_{1} .
\end{gathered}
$$

The control objective is to control the slider $C$ to move periodically. Since $\lambda \rightarrow \lambda_{d}$ means $\left[\begin{array}{ll}f_{1} & f_{2}\end{array}\right]^{T} \rightarrow \mathbf{F}_{\mathbf{d}}$, we chose $\boldsymbol{\theta}_{\mathbf{d}}=4.712 \mathrm{rad}$ and $\boldsymbol{\lambda}_{\mathbf{d}}=15$ in the simulations. The initial position of $x$ is $2.167 \mathrm{~m}$ (i.e. $\theta_{0}=3.1416 \mathrm{rad}$ ) for the slider $C$ and the controlled stroke of the slider $C$ are set to be $0.85 \mathrm{~m}$. Substituting the slider position $x$ into Equations (39)-(41), the crank angle $\theta$ can be obtained.

In the simulations, the responses of the crank angle showing in Figure 7(a) reach the desired value in about $0.9 \mathrm{sec}$. The responses of the position of slider $C$ are shown in Figure 7(b), the associated control efforts $\tau$ are shown in Figures 7(c), and the sliding surface $\mathbf{s}_{1}$ is shown in Figure 7(d). The Lagrange multiplier $\lambda_{C}$ is showed in Figure 8(a). The constraint forces of joints 1-3 are shown in Figures 8(b)-(d), respectively. From Figures 7 and 8, the control objectives of force/motion of the quick-return mechanism are achieved successfully.

\subsection{The toggle mechanism}

For more details of the kinematic and dynamic analysis of the toggle mechanism, refer to [23] and adopt the generalized coordinate vector $\mathbf{Q}=\left[\begin{array}{lll}\theta_{5} & \theta_{2} & \theta_{1}\end{array}\right]^{\mathrm{T}}$ in Equation (1) for the toggle mechanism shown in Figure 3 . The dynamic equation can be obtained and is associated with the following matrices and elements:

$$
\begin{gathered}
\mathbf{M}=\left[\begin{array}{ccc}
A & 0 & E \\
0 & B & H \\
E & H & C_{W}
\end{array}\right] \mathbf{N}_{C}=\left[\begin{array}{ccc}
I & 0 & J \\
0 & K_{W} & L \\
P_{W} & Q_{W} & R_{W}
\end{array}\right] \mathbf{N}_{\mathbf{G}}=0 \\
\mathbf{\Phi}_{\mathbf{Q}}=\left[\frac{\partial \mathbf{\Phi}_{n}(\mathbf{Q})}{\partial \mathbf{Q}_{i}}\right]=\left[\begin{array}{ccc}
0 & r_{3} \cos \theta_{2} & r_{1} \cos \theta_{1} \\
r_{5} \cos \theta_{5} & 0 & r_{4} \cos \left(\theta_{1}+\phi\right)
\end{array}\right] \\
\mathbf{Q}^{\mathbf{A}}=\left[\begin{array}{c}
F_{\mathbf{C}} r_{5} \sin \theta_{5} \\
\left(F_{\mathbf{B}}+F_{\mathbf{E}}\right) r_{1} \sin \theta_{1}+F_{\mathbf{C}} r_{4} \sin \left(\theta_{1}+\phi\right)
\end{array}\right] \\
A=-\frac{1}{2}\left\{\left(\frac{2}{3} m_{5}+2 m_{C} \sin ^{2} \theta_{5}\right) r_{5}^{2}\right\}, B=-\frac{1}{2}\left\{\left(\frac{2}{3} m_{3}+2 m_{B} \sin ^{2} \theta_{2}\right) r_{3}^{2}\right\}, \\
C_{w}=-\frac{1}{2}\left\{\frac{m_{2} r_{1}^{2} r_{4}^{2}}{r_{2}^{2}} \sin ^{2} \phi+2\left(m_{3}+m_{B}\right) r_{1}^{2} \sin ^{2} \theta_{1}+2\left(m_{5}+m_{C}\right) r_{4}^{2} \sin ^{2}\left(\theta_{1}+\phi\right)\right\},
\end{gathered}
$$




$$
\begin{gathered}
E=-\frac{1}{2}\left\{\left(2 m_{C}+m_{5}\right) r_{4} r_{5} \sin \left(\theta_{1}+\phi\right) \sin \theta_{5}\right\}, Q_{W}=-\frac{1}{2}\left\{\left(m_{3}+2 m_{B}\right) r_{1} r_{3} \sin \theta_{1} \cos \theta_{2}\right\} \dot{\theta}_{2}, \\
H=-\frac{1}{2}\left\{\left(2 m_{B}+m_{3}\right) r_{1} r_{3} \sin \theta_{1} \sin \theta_{2}\right\}, I=-\frac{1}{2}\left\{2 m_{C} r_{5}^{2} \sin \theta_{5} \cos \theta_{5}\right\} \dot{\theta}_{5}, \\
J=-\frac{1}{2}\left\{\left(m_{5}+2 m_{C}\right) r_{4} r_{5} \cos \left(\theta_{1}+\phi\right) \sin \theta_{5}\right\} \dot{\theta}_{1}, K_{W}=-\frac{1}{2}\left\{2 m_{B} r_{3}^{2} \sin \theta_{2} \cos \theta_{2}\right\} \dot{\theta}_{2}, \\
L=-\frac{1}{2}\left\{\left(m_{3}+2 m_{B}\right) r_{1} r_{3} \cos \theta_{1} \sin \theta_{2}\right\} \dot{\theta}_{1}, P_{w}=-\frac{1}{2}\left\{\left(m_{5}+2 m_{C}\right) r_{4} r_{5} \sin \left(\theta_{1}+\phi\right) \cos \theta_{5}\right\} \dot{\theta}_{5}, \\
R_{W}=-\frac{1}{2}\left\{2\left(m_{3}+m_{B}\right) r_{1}^{2} \sin \theta_{1} \cos \theta_{1}+2\left(m_{5}+m_{C}\right) r_{4}^{2} \sin \left(\theta_{1}+\phi\right) \cos \left(\theta_{1}+\phi\right)\right\} \dot{\theta}_{1}
\end{gathered}
$$

Where $n=3, m=2$ and $r=1$ are employed in the dynamic analysis.

For the single degree-of-freedom toggle mechanism, there exist two constraint equations as follows:

$$
\mathbf{\Phi}(\mathbf{Q})=\left[\begin{array}{c}
r_{1} \sin \theta_{1}+r_{3} \sin \theta_{2}-f \\
r_{5} \sin \theta_{5}+r_{4} \sin \left(\theta_{1}+\phi\right)-h-f
\end{array}\right]=0 .
$$

where $\phi$ can be obtained by analyzing its geometric relation as:

$$
\phi=\cos ^{-1}\left(\frac{r_{1}^{2}+r_{4}^{2}-r_{2}^{2}}{2 r_{1} r_{4}}\right) .
$$

The positions of sliders B and C can be expressed as follows:

$$
\begin{gathered}
x_{B}=r_{1} \cos \theta_{1}+\left[r_{3}^{2}-\left(f-r_{1} \sin \theta_{1}\right)^{2}\right]^{\frac{1}{2}}, \\
x_{C}=r_{4} \cos \left(\theta_{1}+\phi\right)+\left\{r_{5}^{2}-\left[(h+f)-r_{4} \sin \left(\theta_{1}+\phi\right)\right]^{2}\right\}^{\frac{1}{2}} .
\end{gathered}
$$

The Jacobian matrix of the constraint equation is:

$$
\boldsymbol{\Phi}_{\mathbf{Q}}=\left[\frac{\partial \boldsymbol{\Phi}_{n}(\mathbf{Q})}{\partial \mathbf{Q}_{i}}\right]=\left[\begin{array}{ccc}
0 & r_{3} \cos \theta_{2} & r_{1} \cos \theta_{1} \\
r_{5} \cos \theta_{5} & 0 & r_{4} \cos \left(\theta_{1}+\phi\right)
\end{array}\right] .
$$

Therefore, the matrix defined in Equation (6) is:

$$
\mathbf{L}(\mathbf{q})=\frac{\partial \mathbf{Q}}{\partial \mathbf{q}}=\left[\begin{array}{lll}
\frac{\partial \theta_{5}}{\partial \theta_{1}} & \frac{\partial \theta_{2}}{\partial \theta_{1}} & \frac{\partial \theta_{1}}{\partial \theta_{1}}
\end{array}\right]^{T}=\left[\begin{array}{ccc}
-\frac{r_{4} \cos \left(\theta_{1}+\phi\right)}{r_{5} \cos \theta_{5}} & -\frac{r_{1} \cos \theta_{1}}{r_{3} \cos \theta_{2}} & 1
\end{array}\right]^{T} .
$$

Then, differentiating Equation (52) with respect to time yields: 


$$
\dot{\mathbf{L}}(\mathbf{q})=\left[\begin{array}{c}
\frac{r_{4} r_{5} \dot{\theta}_{1} \sin \left(\theta_{1}+\phi\right) \cos \theta_{5}-r_{4} r_{5} \dot{\theta}_{5} \sin \theta_{5} \cos \left(\theta_{1}+\phi\right)}{r_{5}^{2} \cos ^{2} \theta_{5}} \\
\frac{r_{1} r_{3} \dot{\theta}_{1} \sin \theta_{1} \cos \theta_{2}-r_{1} r_{3} \dot{\theta}_{2} \sin \theta_{2} \cos \theta_{1}}{r_{3}^{2} \cos ^{2} \theta_{2}} \\
0
\end{array}\right]=\left[\begin{array}{c}
\dot{L}_{11} \\
\dot{L}_{21} \\
0
\end{array}\right] .
$$

The dynamic equation of the toggle mechanism, when restricted to the constraint equation (51), can be expressed as:

$$
\begin{aligned}
& {\left[\begin{array}{c}
A \times\left\{-\frac{r_{4} \cos \left(\theta_{1}+\phi\right)}{r_{5} \cos \theta_{5}}\right\}+E \\
B \times\left\{-\frac{r_{1} \cos \theta_{1}}{r_{3} \cos \theta_{2}}\right\}+H \\
E \times\left\{-\frac{r_{4} \cos \left(\theta_{1}+\phi\right)}{r_{5} \cos \theta_{5}}\right\}+A \times\left\{-\frac{r_{1} \cos \theta_{1}}{r_{3} \cos \theta_{2}}\right\}+C_{W}
\end{array}\right] \ddot{\theta}_{1}+} \\
& {\left[\begin{array}{c}
A \times \dot{L}_{11}+I \times\left\{-\frac{r_{4} \cos \left(\theta_{1}+\phi\right)}{r_{5} \cos \theta_{5}}\right\}+J \\
B \times \dot{L}_{21}+K_{W} \times T+L \\
E \times \dot{L}_{11}+H \times \dot{L}_{21}+P_{W} \times\left\{-\frac{r_{4} \cos \left(\theta_{1}+\phi\right)}{r_{5} \cos \theta_{5}}\right\}+Q_{W} \times\left\{-\frac{r_{1} \cos \theta_{1}}{r_{3} \cos \theta_{2}}\right\}+R_{W}
\end{array}\right] \dot{\theta}_{1}} \\
& =\left[\begin{array}{c}
F_{\mathbf{C}} r_{5} \sin \theta_{5} \\
\left(F_{\mathbf{B}}+F_{\mathbf{E}}\right) r_{3} \sin \theta_{2} \\
\left(F_{\mathbf{B}}+F_{\mathbf{E}}\right) r_{1} \sin \theta_{1}+F_{\mathbf{C}} r_{4} \sin \left(\theta_{1}+\phi\right)
\end{array}\right]+\left[\begin{array}{l}
u_{1} \\
u_{2} \\
u_{3}
\end{array}\right]+\left[\begin{array}{cc}
0 & r_{5} \cos \theta_{5} \\
r_{3} \cos \theta_{2} & 0 \\
r_{1} \cos \theta_{1} & r_{4} \cos \left(\theta_{1}+\phi\right)
\end{array}\right]\left[\begin{array}{l}
\lambda_{1} \\
\lambda_{2}
\end{array}\right] .
\end{aligned}
$$

The parameters $A, E, B, H, C_{W}, \dot{L}_{11}, \dot{L}_{21}, I, J, K_{W}, T, L, P_{W}, Q_{W}$ and $R_{W}$ are shown in Equation (46). It is noted that $F_{B}$ is the friction force, $F_{E}$ is the external force acting on the slider $\mathrm{B}, F_{C}$ is the applied force acting on the slider $\mathrm{C}$, and $f_{1}=r_{5} \lambda_{2} \cos \theta_{5}, f_{2}=r_{3} \lambda_{2} \cos \theta_{2}$ and $f_{3}=r_{1} \lambda_{1} \cos \theta_{1}+r_{4} \lambda_{2} \cos \left(\theta_{1}+\phi\right)$ are the constraint forces.

The control objective is to regulate the position of slider B moving from the left to the right ends. The initial position of $X_{B}$ is $0.104 \mathrm{~m}\left(\right.$ i.e. $\left.\theta_{2}\left(t_{0}\right)=4.712 \mathrm{rad}\right)$, and its expected position is $0.114 \mathrm{~m}$. The desired values are $\boldsymbol{\theta}_{d}\left(t_{f}\right)=5.76 \mathrm{rad}$ and $\lambda_{d}=15$ in the simulations. Furthermore, in order to show that the SMC is insensitive to parametric variation, the effects of friction forces in joints are considered in this toggle mechanism system by using the Lagrange multiplier method.

The comparisons between the nominal case without considering friction forces and the case with friction forces are shown in Figures 9-11. Figures 9(a)-(c) show the trajectories of angles $\theta_{1}, \theta_{2}$ and $\theta_{5}$, respectively. Figures $10(\mathrm{a})-(\mathrm{b})$ show the positions of sliders $\mathrm{B}$ and $\mathrm{C}$, respectively. Figures 10(c)-(d) illustrate the control effort $\tau$ and the sliding surface $s_{1}$, respectively. Finally, Figure 11(a) shows the Lagrange multiplier $\lambda_{C}$ and Figures 11(b)-(d) address the constraint forces $f_{1}, f_{2}$ and $f_{3}$ acting on the joints 1,2 , and 3 , respectively.

From the numerical results, it is found that the control efforts $\tau$ are almost identical for both cases whether the friction forces are considered or not. From the above figures, the 
force/motion control objective of a toggle mechanism using the SMC is achieved successfully and the system responses are insensitive to the effects of friction forces.

\section{Conclusion}

Based on the Lyapunov theorem, we successfully derived a generalized SMC algorithm in a simple manner. The algorithm used tracking the Lagrange multiplier error to facilitate controller design and proposed a separate sliding surface in terms of the displacement and velocity. Furthermore, some properties of the dynamic structure were presented and used to reduce the dynamic model equations. Finally, the slider-crank, quick-return, and toggle mechanisms were employed to illustrate and verify the methodology developed. From the numerical results, we conclude that the effectiveness in application of the developed SMC method is successfully verified in regards to the force/motion controls for these three typical mechanisms. First, fast attainment of the control objective: in the simulations, the three typical mechanisms reached the desired value in less than 1 second. Second, no overshoot in the control process: from the numerical results, the force/motion control objectives of the three mechanisms using the SMC were achieved successfully and the system responses did not overshoot in the whole control process. Third, insensitivity to parametric variation: the control efforts $\tau$ are almost identical and the system responses are insensitive to the effects of the friction forces.

\section{Acknowledgment}

The authors would like to thank Nation Science Council of Republic of China, Taiwan, for financial support of this research under contract no. NSC 98-2221-E-327 -010 -MY3.

\section{Appendix A}

Define the matrix $N(Q, \dot{Q})=\dot{M}(Q)-2 N_{C}(Q, \dot{Q})$, then $N(Q, \dot{Q})$ is skew symmetric, i.e., the components $n_{j k}$ of $N$ satisfy $n_{j k}=-n_{k j}$.

Proof: Given the inertia matrix $M(Q)$, the $k j^{\text {th }}$ component of $\dot{M}(Q)$ is given by the chain rule as

$$
\dot{m}_{k j}=\sum_{i=1}^{n} \frac{\partial m_{k j}}{\partial Q_{i}} \dot{Q}_{i}
$$

and the $k j^{\text {th }}$ component of $N(Q, \dot{Q})=\dot{M}(Q)-2 N_{C}(Q, \dot{Q})$ is given by

$$
\begin{aligned}
& \dot{n}_{k j}=\dot{m}_{k j}-2 c_{k j} \\
& =\sum_{i=1}^{n}\left[\frac{\partial m_{k j}}{\partial Q_{i}}-\left\{\frac{\partial m_{k j}}{\partial Q_{i}}+\frac{\partial m_{k i}}{\partial Q_{i}}-\frac{\partial m_{i j}}{\partial Q_{k}}\right\} \dot{Q}_{i}\right]=\sum_{i=1}^{n}\left[\frac{\partial m_{i j}}{\partial Q_{k}}-\frac{\partial m_{k i}}{\partial Q_{j}}\right] \dot{Q}_{i}
\end{aligned}
$$

Since the inertia matrix $M(Q)$ is symmetric, i.e., $m_{i j}=m_{j i}$, it follows from Equation (A.2), the $j k^{\text {th }}$ component of $\left.N(Q, \dot{Q})\right)$ is

$$
n_{j k}=\sum_{i=1}^{n}\left[\frac{\partial m_{i j}}{\partial Q_{j}}-\frac{\partial m_{k i}}{\partial Q_{k}}\right] \dot{Q}=-n_{k j} .
$$




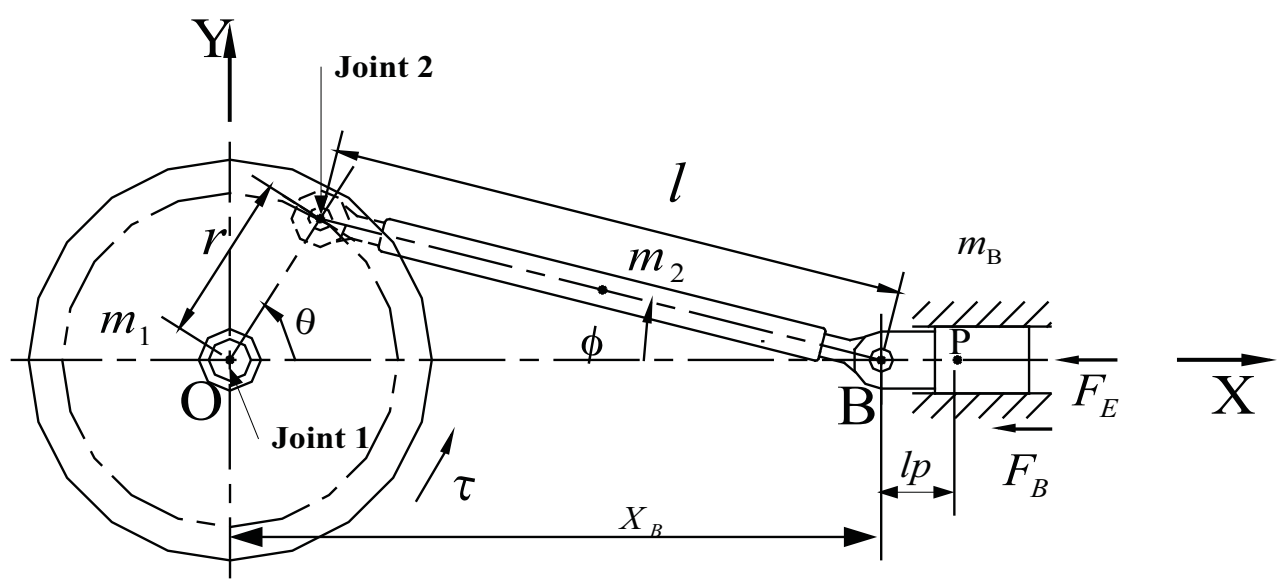

Fig. 1. The physical model of the slider-crank mechanism 


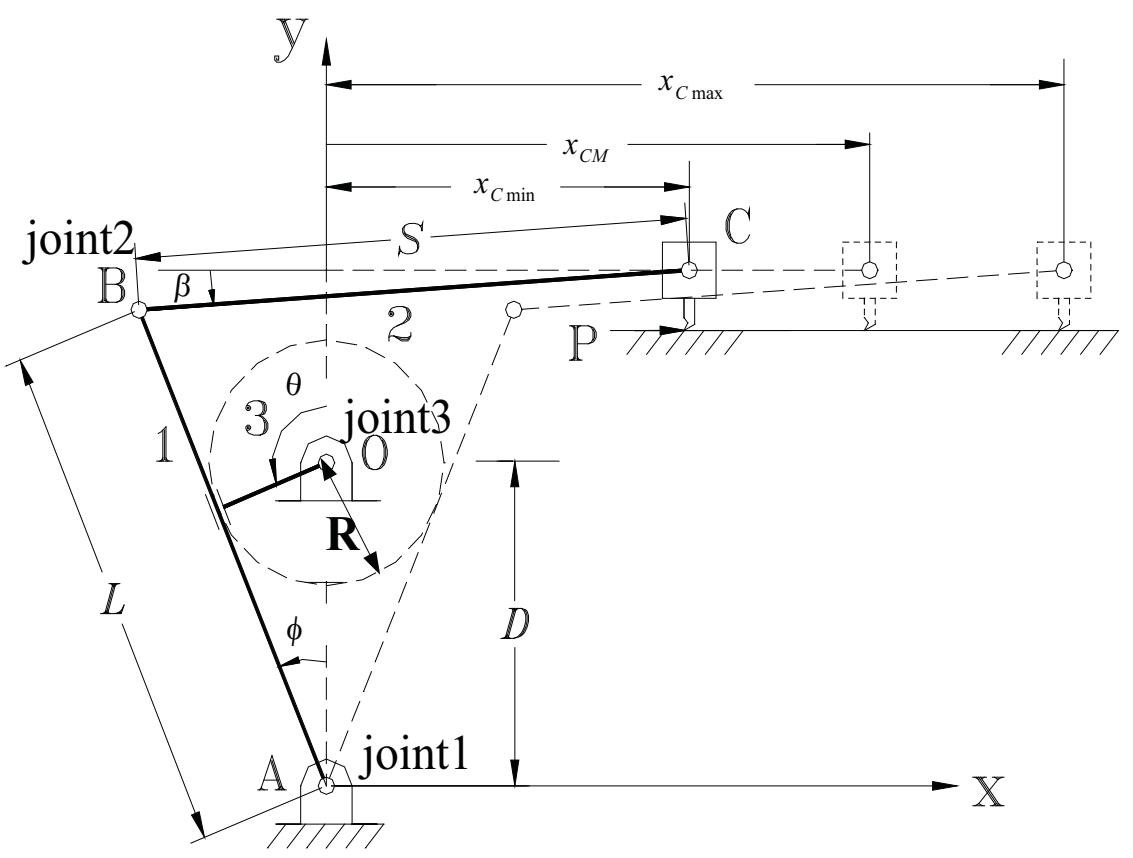

Fig. 2. The physical model of the quick-return mechanism 


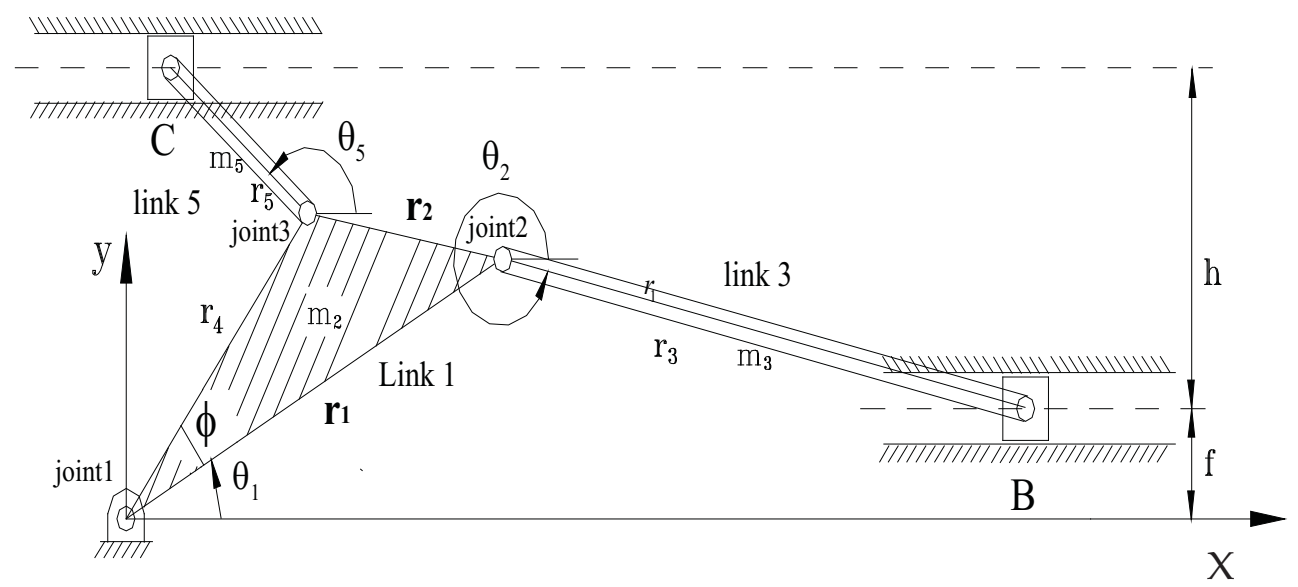

Fig. 3. The physical model of the toggle mechanism.

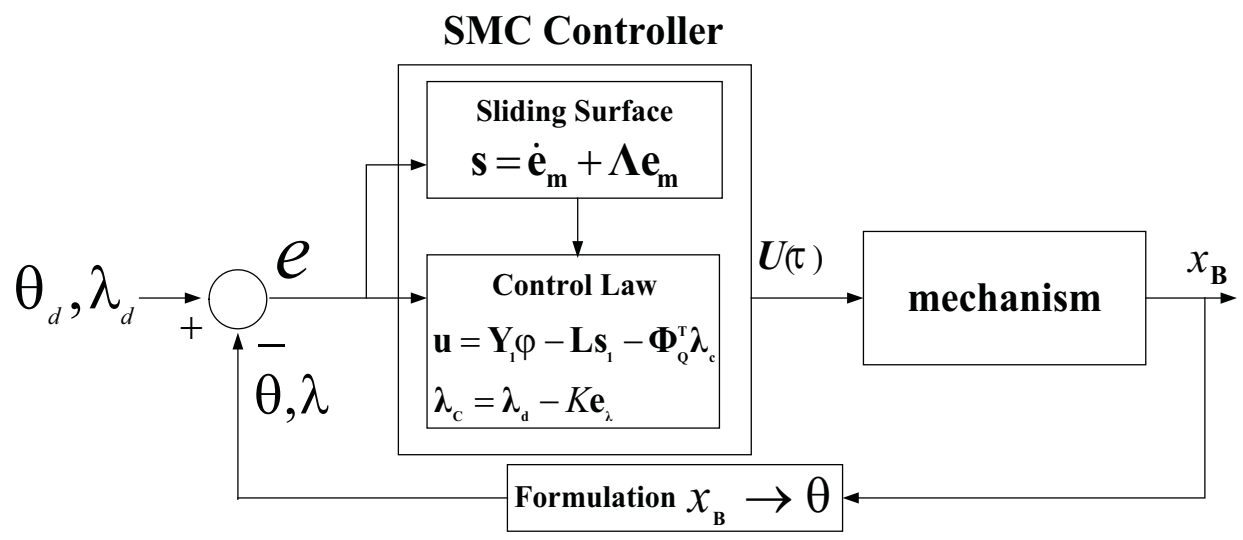

Fig. 4. The block diagram of mechanisms using the SMC controller 

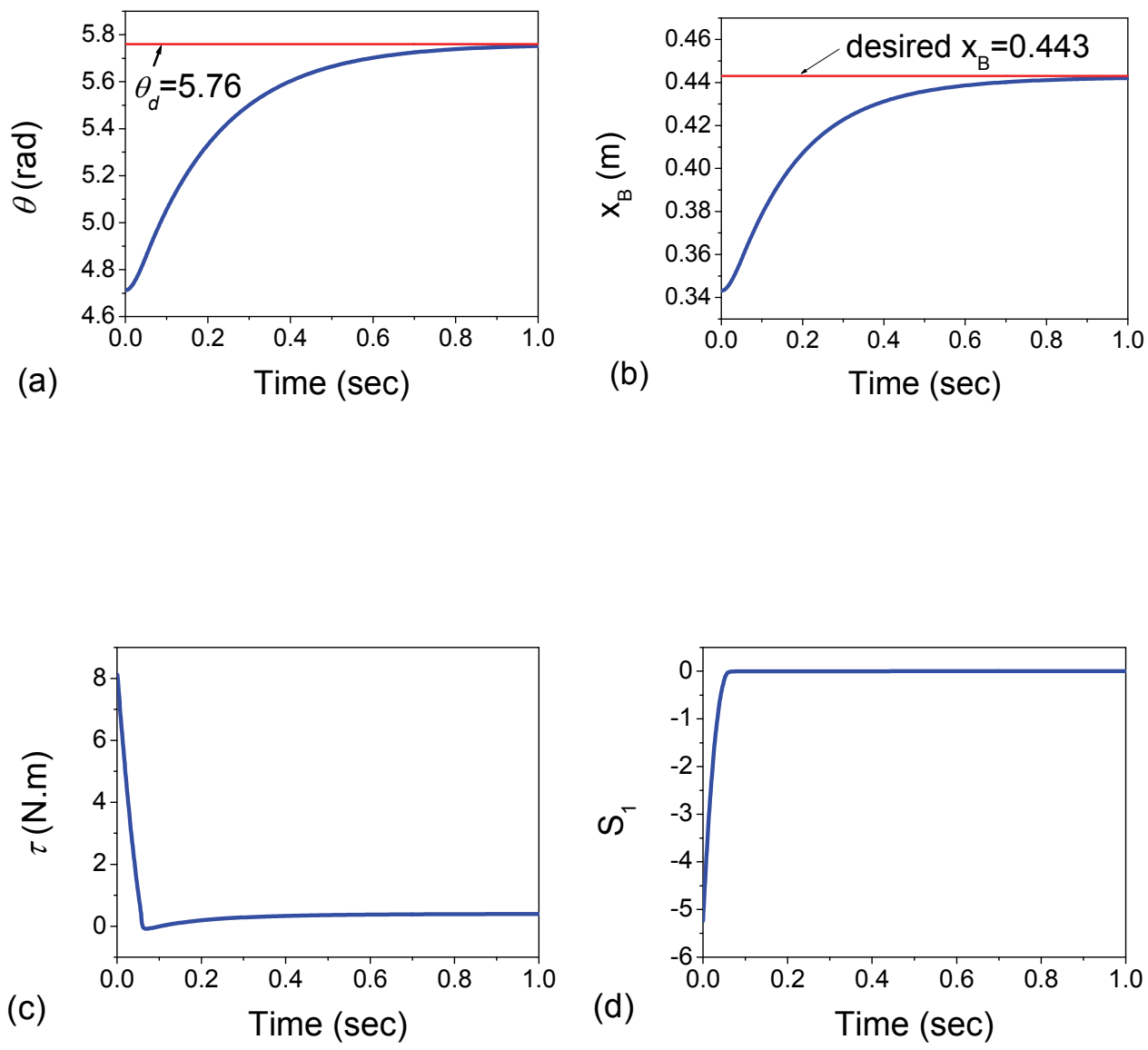

Fig. 5. The simulation results of the slider-crank mechanism. ('-'desired value; '-'actual trajectory) (a) Response trajectories of the crank angle $\theta$. (b) Response trajectories of the slider B in position $X_{B}$. (c) Response trajectories of the control effort $\tau$. (d) Response trajectories of the sliding surface $s_{1}$. 

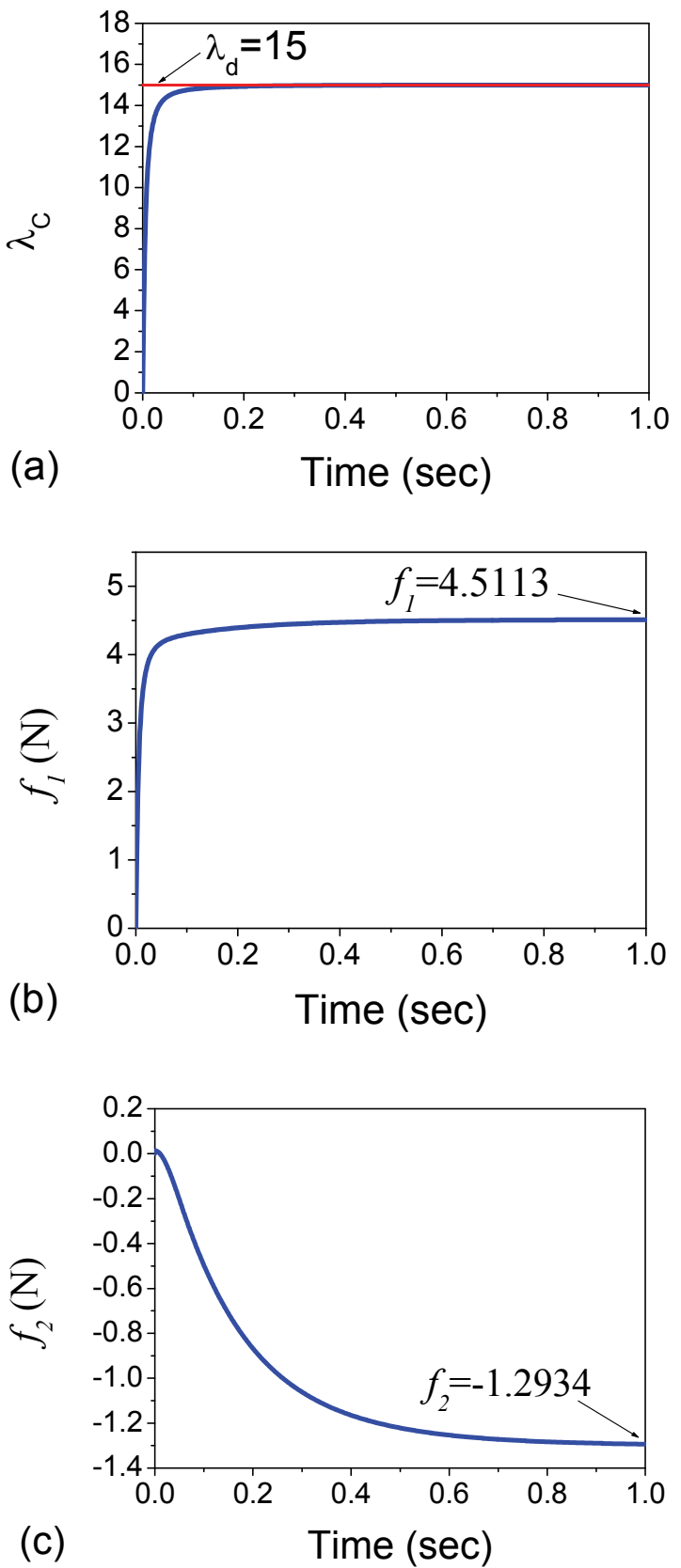

Fig. 6. The simulation results of the slider-crank mechanism. ('-'desired value; '-'actual trajectory) (a) Response trajectories of the Lagrange multiplier $\lambda_{C} \cdot(b)$ Response trajectories of the constraint force $f_{1}$. (c) Response trajectories of the constraint force $f_{2}$. 

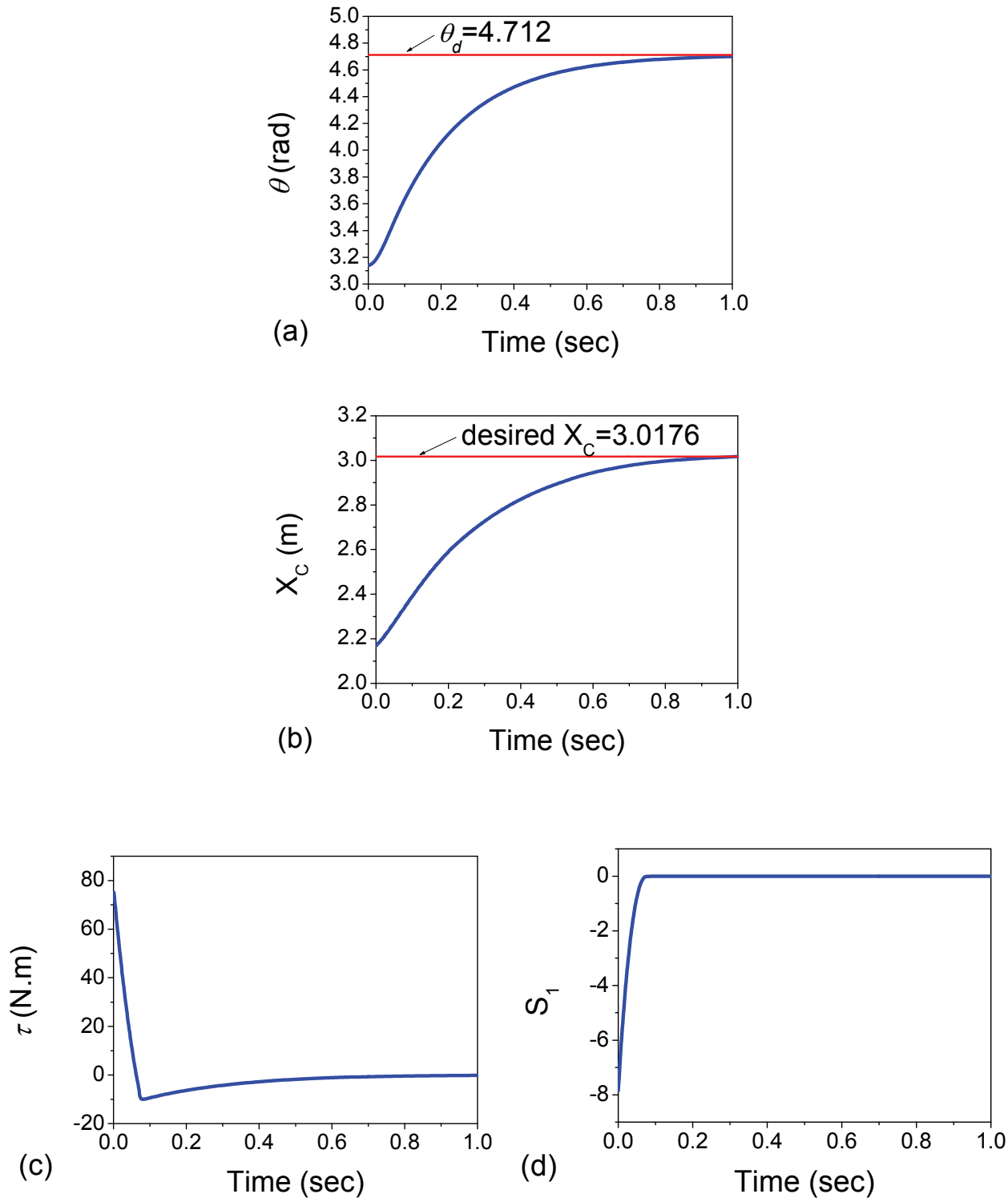

Fig. 7. The simulation results of the quick-return mechanism. ('-'desired value; '-'actual trajectory) (a) Response trajectories of the crank angle displacement $\theta$. (b) Response trajectories of the slider $C$ in position $X_{C}$. (c) Response trajectories of the control effort $\tau$. (d) Response trajectories of the sliding surface $s_{1}$. 

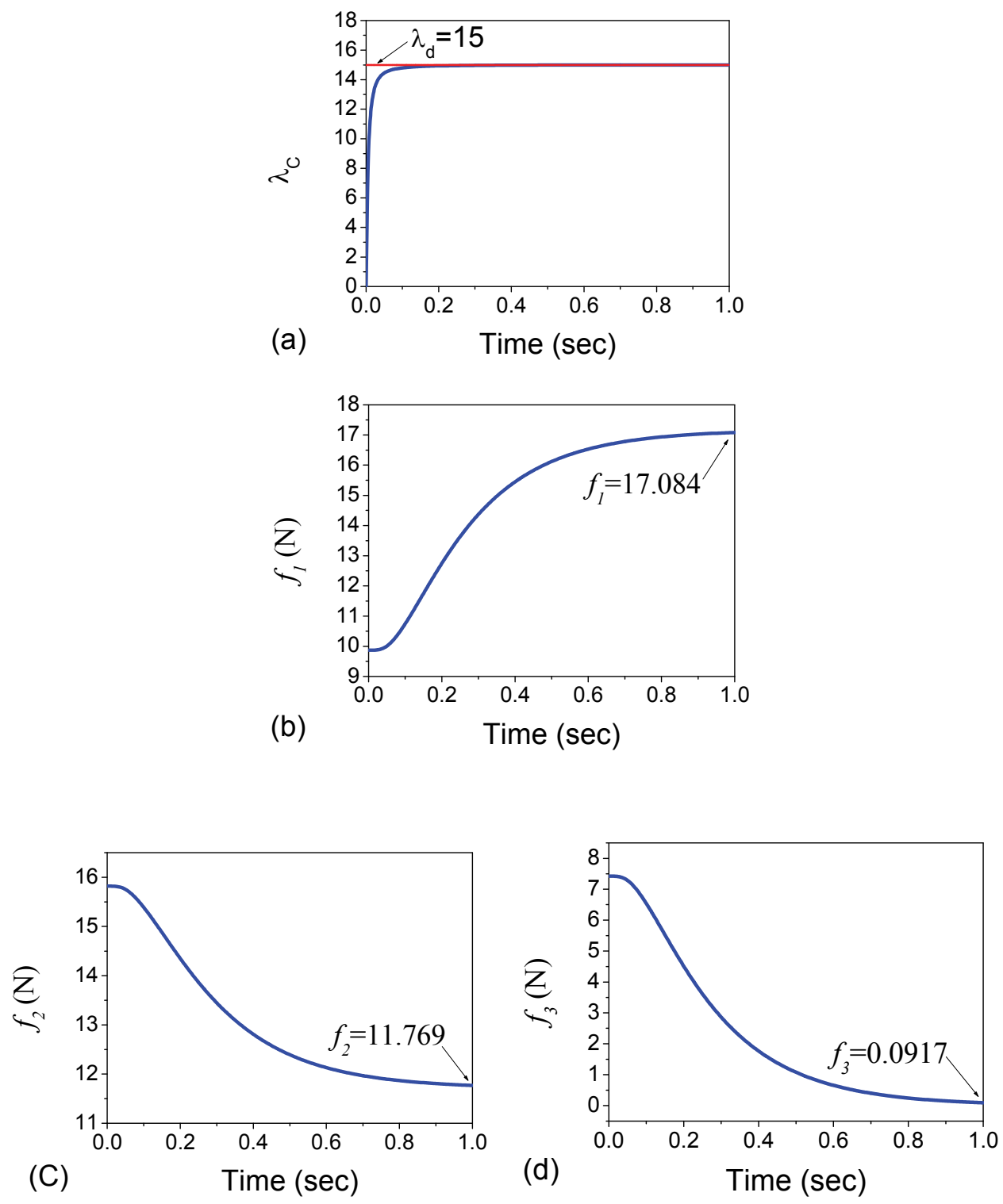

Fig. 8. The simulation results of the quick-return mechanism. ('-'desired value; '-'actual trajectory) (a) Response trajectories of the Lagrange multiplier $\lambda_{C} \cdot(b)$ Response trajectories of the constraint force $f_{1}$. (c) Response trajectories of the constraint force $f_{2}$. (d) Response trajectories of the constraint force $f_{3}$. 

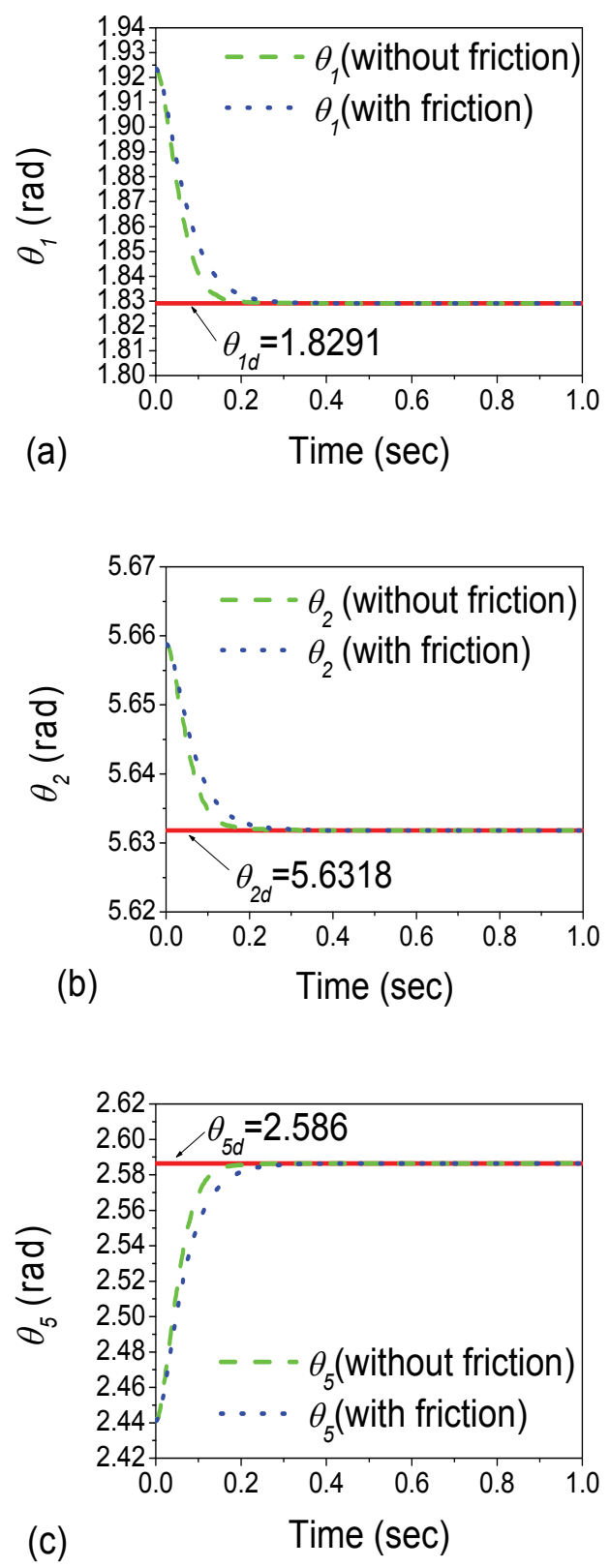

Fig. 9. The simulation results of the toggle mechanism. ('-'desired curve; '---'actual trajectory (without friction), '---'actual trajectory (with friction and $f_{r}=0.3$ )) (a) Response trajectories of the angle displacement $\theta_{1}$. (b) Response trajectories of the angle displacement $\theta_{2}$. (c) Response trajectories of the angle displacement $\theta_{5}$. 

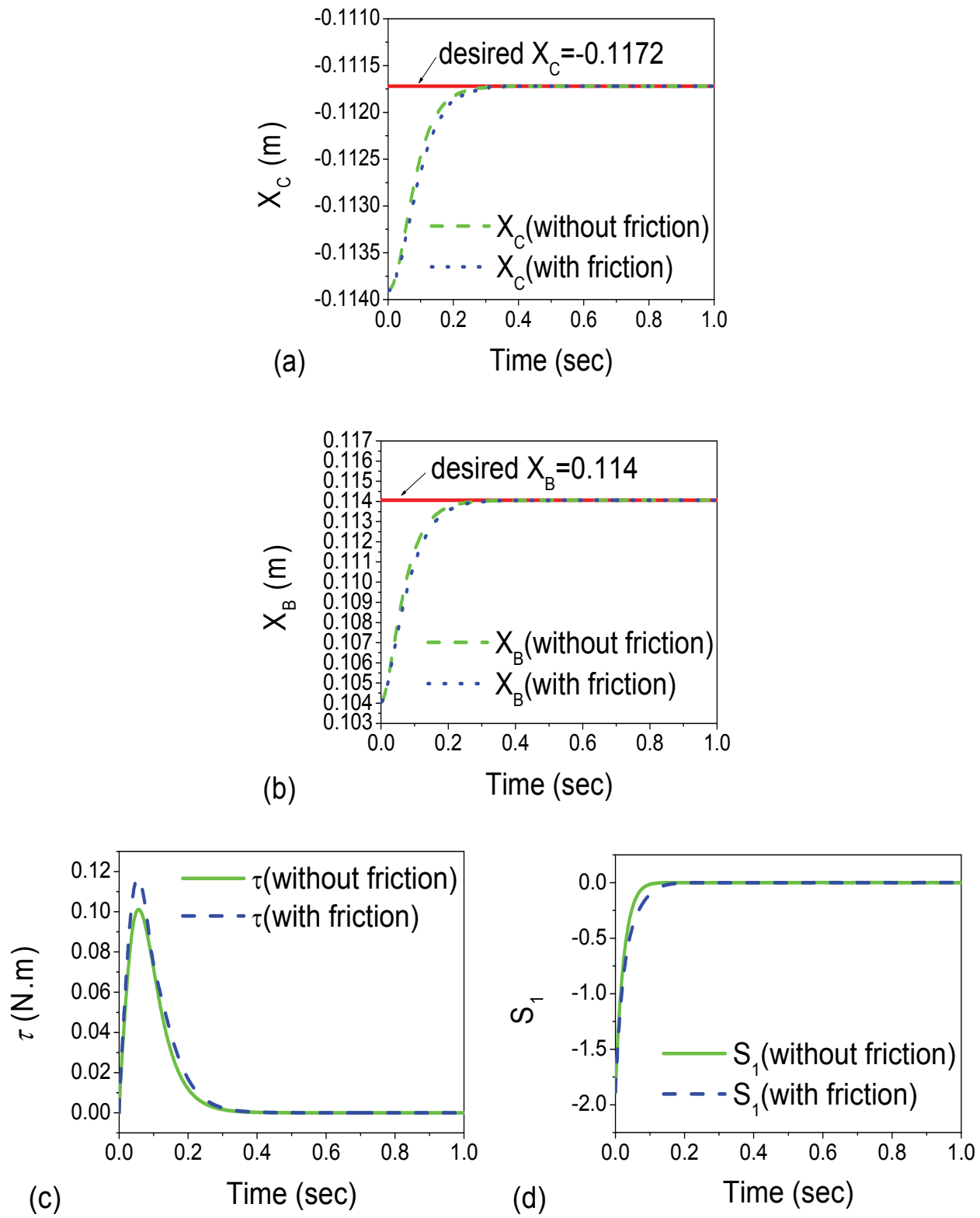

Fig. 10. The simulation results of the toggle mechanism. ('-'desired curve; '--' actual trajectory (without friction ), '---'actual trajectory (with friction and $f_{r}=0.3$ )) (a) Response trajectories of the slider $C$ in position $X_{C}$. (b) Response trajectories of the slider B in position $X_{B}$. (c) Response trajectories of the control effort $\tau$. (d) Response trajectories of the sliding surface $s_{1}$. 

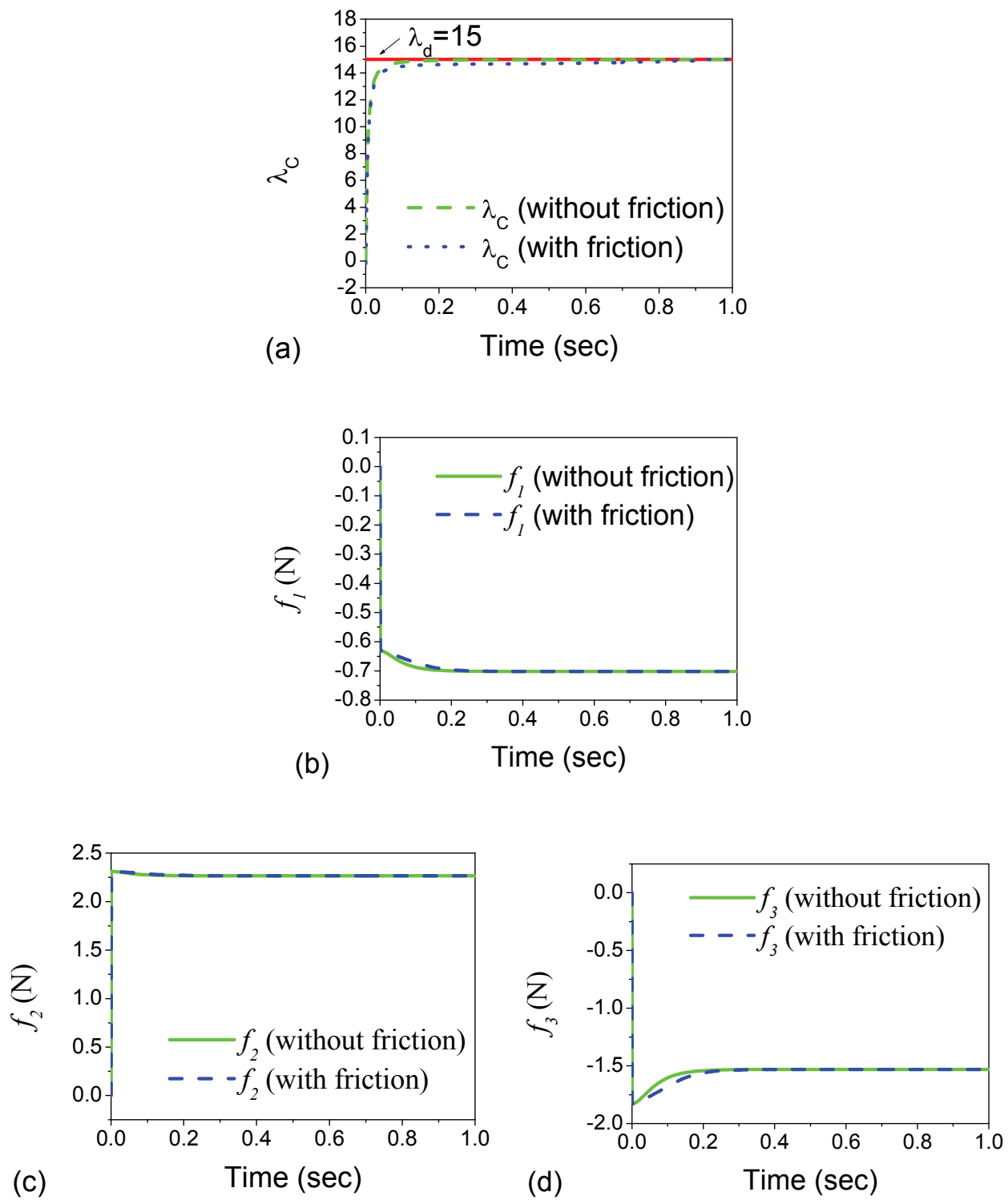

Fig. 11. The simulation results of the toggle mechanism. ('-'desired curve; '---'actual trajectory (without friction), '---'actual trajectory (with friction and $f_{r}=0.3$ )) (a) Response trajectories of the Lagrange multiplier $\lambda_{C}$. (b) Response trajectories of the constraint force $f_{1}$. (c) Response trajectories of the constraint force $f_{2}$. (d) Response trajectories of the constraint force $f_{3}$ 


\section{References}

[1] Fung, R. F., "Dynamic Analysis of the Flexible Connecting Rod of a Slider-Crank Mechanism," ASME Journal of Vibration and Acoustic, Vol. 118, No. 4, pp. 687689(1996).

[2] Fung, R. F., and Chen, H. H., "Steady-State Response of the Flexible Connecting Rod of a Slider-Crank Mechanism with Time-Dependent Boundary Condition," Journal of Sound and Vibration, Vol. 199, No. 2, pp. 237-251(1997).

[3] Fung, R. F., "Dynamic Response of the Flexible Connecting Rod of a Slider-Crank Mechanism with Time-Dependent Boundary Effect," Computer \& Structure, Vol. 63, No. 1, pp. 79-90(1997).

[4] Fung, R. F., Huang, J. S., Chien, C. C., and Wang, Y. C., “Design and Application of a Continuous Repetitive Controller for Rotating Mechanisms," International Journal of Mechanical Sciences, Vol. 42, pp. 1805-1819(2000).

[5] Lin, F. J., Fung, R. F., and Lin Y. S., "Adaptive Control of Slider-Crank Mechanism Motion: Simulations and Experiments," International Journal of Systems Science, Vol. 28, No. 12, pp. 1227-1238(1997).

[6] Lin, F. J., Lin, Y. S. and Chiu, S. L., "Slider-Crank Mechanism Control using Adaptive Computed Torque Technique," Proceedings of the IEE Control Theory Application, Vol. 145, No. 3, pp. 364-376(1998).

[7] Lin, F. J., Fung, R. F., Lin, H. H., and Hong, C. M., "A Supervisory Fuzzy Neural Network Controller for Slider-Crank Mechanism," Proceedings of the IEEE Control Applications Conferences, pp. 1710-1715(1999).

[8] Utkin, V. I., Sliding Modes and Their Applications, Mir: Moscow (1978).

[9] Utkin, V. I., "Discontinuous Control System: State of the Art in Theory and Application," Preprint 10th IFAC World Congress, Vol. 1, pp. 75(1987).

[10] Compere, M. D. and Longoria, R. G., "Combined DAE and Sliding Mode Control Methods for Simulation of Constrained Mechanical System," ASME Journal of Dynamic System, Measurement, and Control, Vol. 122, pp. 691-698(2000).

[11] Su, C. Y., Leung, T. P., and Zhou, Q. J., "Force/Motion Control of Constrained Robots Using Sliding Mode," IEEE Transactions on Automatic Control, Vol. 37, No. 5, pp. 668-672(1992).

[12] Grabbe, M. T., and Bridges, M. M., "Comments on "Force/Motion Control of Constrained Robots Using Sliding Mode", " IEEE Transactions on Automatic Control, Vol. 39, No. 1, pp. 179(1994).

[13] Slotine, J. J. E. and Li, W., Applied Nonlinear Control. Englewood Cliffs, NJ: Prentice-Hall (1991).

[14] Lian, K. Y. and Lin, C. R., “Sliding Mode Motion/Force Control of Constrained Robots," IEEE Transactions on Automatic Control, Vol. 43, No. 8, pp. 1101-1103(1998).

[15] Dixon, W. E. and Zergeroglu, E., "Comments on "Sliding Mode Motion/Force Control of Constrained Robots"," IEEE Transactions on Automatic Control, Vol. 45, No. 8, pp. 1576(2000).

[16] Fung, R. F., Shue, L. C, "Regulation of a Flexible Slider-Crank Mechanism by Lyapunov's Direct Method," Mechatronics, Vol. 12, pp. 503-509(2002).

[17] Fung, R. F., Sun, J. H, “Tracking Control of the Flexible Slider-Crank Mechanism System Under Impact," Journal of Sound and Vibration, Vol. 255, pp. 337-355(2002). 
[18] McClamroch, N. H., and Wang, D. W., "Feedback Stabilization and Tracking of Constrained Robots," IEEE Transactions on Automatic Control, Vol. 33, No. 5, pp. 419426(1988).

[19] Fung, R. F., Lin, F. J., Huang, J. S., and Wang, Y. C., “Application of Sliding Mode Control with A Low Pass Filter to the Constantly Rotating Slider-Crank Mechanism," The Japan Society of Mechanical Engineering, Series C, Vol. 40, No. 4, pp. 717-722(1997).

[20] Parviz, E. N., Computer-Aided Analysis of Mechanical System. Prentice-Hall, Englewood Cliffs NJ (1988).

[21] Fung, R. F. and Chen, K. W., "Constant Speed Control of the Quick-return Mechanism," The Japan Society of Mechanical Engineering, Series C, Vol. 40, No. 3, pp. 454461(1997).

[22] Fung, R. F. and Yang, R. T., "Motion control of an electrohydraulic actuated toggle mechanism," Mechatronics, Vol. 11, pp. 939-946(2001).

[23] Fung, R. F., Wu, J. W. and Chen, D. S., “A variable structure control toggle mechanism driven by a linear synchronous motor with joint coulomb friction," Journal of sound and vibration, Vol. 274, No. 4, pp. 741-753(2001).

[24] Slotine, J. J. E. and Sastry, S. S., "Tracking control of nonlinear system using sliding surface with application to robot manipulators," International journal of control, Vol. 38, pp. 465-492(1983). 


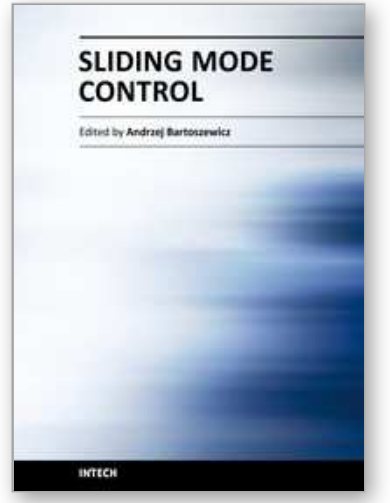

\author{
Sliding Mode Control \\ Edited by Prof. Andrzej Bartoszewicz
}

ISBN 978-953-307-162-6

Hard cover, 544 pages

Publisher InTech

Published online 11, April, 2011

Published in print edition April, 2011

The main objective of this monograph is to present a broad range of well worked out, recent application studies as well as theoretical contributions in the field of sliding mode control system analysis and design. The contributions presented here include new theoretical developments as well as successful applications of variable structure controllers primarily in the field of power electronics, electric drives and motion steering systems. They enrich the current state of the art, and motivate and encourage new ideas and solutions in the sliding mode control area.

\title{
How to reference
}

In order to correctly reference this scholarly work, feel free to copy and paste the following:

Rong-Fong Fung and Chin-Fu Chang (2011). Force/Motion Sliding Mode Control of Three Typical Mechanisms, Sliding Mode Control, Prof. Andrzej Bartoszewicz (Ed.), ISBN: 978-953-307-162-6, InTech, Available from: http://www.intechopen.com/books/sliding-mode-control/force-motion-sliding-mode-control-ofthree-typical-mechanisms

\section{INTECH}

open science | open minds

\section{InTech Europe}

University Campus STeP Ri

Slavka Krautzeka 83/A

51000 Rijeka, Croatia

Phone: +385 (51) 770447

Fax: +385 (51) 686166

www.intechopen.com

\section{InTech China}

Unit 405, Office Block, Hotel Equatorial Shanghai

No.65, Yan An Road (West), Shanghai, 200040, China

中国上海市延安西路 65 号上海国际贵都大饭店办公楼 405 单元

Phone: +86-21-62489820

Fax: +86-21-62489821 
(C) 2011 The Author(s). Licensee IntechOpen. This chapter is distributed under the terms of the Creative Commons Attribution-NonCommercialShareAlike-3.0 License, which permits use, distribution and reproduction for non-commercial purposes, provided the original is properly cited and derivative works building on this content are distributed under the same license. 